\title{
On the Structure of Turbulence in a Low- Speed Axial Fan with Inlet Guide Vanes
}

\author{
Fernández Oro, J.M., Argüelles Díaz, K.M., \\ Santolaria Morros, C.; Blanco Marigorta, E. \\ Universidad de Oviedo, Área de Mecánica de Fluidos. \\ Campus de Viesques, 33271, Gijón (Asturias), Spain. \\ jesusfo@uniovi.es
}

\begin{abstract}
This paper analyzes the structure of turbulence in a single stage, low-speed axial fan with inlet guide vanes. Turbulence intensity values and integral length scales have been obtained using hot-wire anemometry for three different operating points and two different axial gaps between the stator and the rotor. These measurements were carried out in two transversal sectors, one between the rows and the other rotor downstream, covering the whole span of the stage for a complete stator pitch. Since total unsteadiness is composed of the contribution of both periodic and random unsteadiness, a processing data method was developed to filter deterministic unsteadiness in the raw velocity traces. Velocity signals were transformed into the frequency domain by removing all the contributions coming from the rotational frequency, the blade passing frequency and its harmonics. Consequently, coherent flow structures were decoupled and thus background levels of turbulence-RMS values of random fluctuations- were determined across the stage. Additionally, this unsteady segregation revealed further information about the transport of the turbulent structures in the unsteady, deterministic flow patterns. Therefore, anisotropic turbulence, generated at the shear layers of the wakes, could be identified as the major mechanism of turbulence generation, rather than free-stream, nearly isotropic turbulence of wake-unaffected regions. Finally, spectra and autocorrelation analysis of random fluctuations were also used to estimate integral length scales -larger eddy sizes- of turbulence, providing insight on the complete picture of the turbulent flow.
\end{abstract}

\section{INTRODUCTION}

Total unsteadiness in a multistage environment is a key parameter in the performance of any axial turbomachine. In case of axial compressors, much effort has been focused on understanding the boundary layer transition from laminar to turbulent on blade surfaces (Henderson et al., [1]). The vortical disturbances that are created by wakes convected from blade rows further upstream may lead to boundary layer transition. This periodic impinging of incoming wakes onto the blades is a well-known "wake-induced" transition. In addition, a high level of turbulence, rather than those periodic disturbances on the blade surfaces, can also be responsible for the turbulent shear layer to be set on (Mailach et al., [2]). Therefore, in order to achieve a good description for both mechanisms, it is necessary to segregate its relative influence on the development of the unsteady boundary layers on the blades.

The flow field inside a turbomachine is characterized by its complex unsteadiness. When this unsteadiness is considered as a whole unique fluctuation, this total variation leads to the establishment of the classical Reynolds stresses into the mean flow.
However, the total unsteadiness can also be considered as the contribution of both periodic and random components. The periodic fluctuation, usually known as "unsteadiness", consists of all nonuniformities and unsteadiness clocked with the shaft speed and the blade passing frequency (BPF). It can be addressed by means of an average-passage technique, in which the NavierStokes equations are transformed into a set of steady, full 3-D viscous equations, separately for each row (Uzol et al., [3]). As a result, the effects of phasedependent unsteadiness are accounted for through additional terms in the equations, known as "deterministic stresses". Its solution provides the average-passage flow, which is a time-averaged flow field, periodic over the pitch of the blade row of interest. On the other hand, the closure problem is completed with those remaining stresses due to the random and chaotic component. Actually, the Reynolds stresses appear in the final equations taking into account just purely stochastic fluctuations, referred to as "turbulence".

The segregation of "unsteadiness" from "turbulence" in multistage turbomachinery can be easily observed in any velocity trace, rather than in the complex, non- 
linear set of Navier-Stokes equations. Previous works have dealt with this idea, using velocity traces obtained through hot-wire anemometry (Camp et al., [4]), or even pressure signals from rapid response transducers (Cherret et al., [5]). The simplest method to remove periodic fluctuations consists in ensemble-averaging the velocity trace and then subtracting this processed signal from the original data ([1], [3], [5]). Unfortunately, though this is an exact definition which gives useful results, it is just filtering all the unsteadiness related to the BPF. Other "large-scale" unsteadiness with periodic features like vortex shedding, unsteadiness of separation points, misalignment of the blades or fluttering of separated flows is not removed by using this method. In practice, this is because there is no exact frontier separating the periodic unsteadiness of large-scale fluctuations from the large-scale eddies of random turbulence. Consequently, it is also necessary to include some other filtering procedure to remove clearly the effects of all the periodic unsteadiness from the raw data. Some authors have defined a cut-off frequency to extract just "small-scale" fluctuations (Goto [6], with single hot-wire anemometry, and Sentker et al. [7-8], with dual hot-wire probes), neglecting much of the turbulent energy of large-scale eddies. In order to avoid this inconvenience, a method with a frequency domain basis is used to identify peaks (at the rotational frequency, at the BPF and all the relevant harmonics) in the Fourier transform of the signal. These peaks, representing periodic events, are digitally filtered out by setting its amplitudes to zero. The truncated power spectrum is then transformed back into the time domain to give the turbulent signal ([4], Ballesteros et al., [9]).

This paper analyzes the structure of turbulent data, obtained using hot-wire anemometry, in a single-stage, low-speed axial fan with inlet guide vanes. Up to now, only total unsteadiness had been treated in detail in the case of industrial axial fans (Velarde-Suárez et al., [10]). In this work, a digital filtering procedure in the frequency domain (adapted from [5]) has been employed to segregate random fluctuations from periodic disturbances downstream of the rotor. As a result, distributions and maps of turbulence intensity are presented between the rows and downstream of the rotor. In addition, the transport of turbulent structures in the unsteady deterministic flow has been analyzed in temporal diagrams. The impact of the flow coefficient in the dispersion of turbulent wakes shed from the stator is also considered, and phase-averaged representations of the turbulence in the rotor wakes are shown as a perfect picture of the structure of turbulence. Finally, an estimation of the integral length scales across the stage (taken from [5] and Huyer et al., [11]) is presented as useful data for CFD applications, since it gives valuable information about those larger eddies, which may be resolved instead of being modeled.

\section{EXPERIMENTAL SETUP AND PROCEDURES}

Experimental Facility and Low-Speed Axial Fan. The experimental measurements of the unsteady flow field have been conducted in a single stage, low-speed axial fan with a stator-rotor configuration. It is operated in an open-loop facility that includes a regulation cone at the inlet for flow rate control and a Venturi nozzle to obtain accurate measurements of the flow rate (according to normative BS 848). A long duct of twenty fan diameters length, placed between the control valve and the fan bell-mouth inlet, assures uniform flow at the fan intake.

The fan analyzed presents a nine-blade rotor based on the NACA 65 family, and designed in order to provide a free-vortex distribution of the absolute tangential velocity component. The flow is previously guided by a thirteen-vane stator based on the British circular family $\mathrm{C} 1$. The hub and tip diameters of the machine are 380 and $820 \mathrm{~mm}$ respectively, giving a hub-to-tip ratio of 0.46 . The tip clearance is $2.3 \%$ of the blade height. Basic geometrical parameters of vanes and blades at hub, midspan and tip sections are summarized in table 1.

The fan allows for the manual changing of the axial gap between the rows, by means of a set of annular rings that can be removed or added to increase or decrease the stator-rotor separation. In these experiments, two axial gap configurations were tested: a larger, nominal gap of a $50 \%$ of the blade chord, and a lower gap of a $37 \%$ of the blade chord, both at the hub section. The fan, rotating at $2400 \mathrm{rpm}$, is driven by an AC $37 \mathrm{~kW}$ motor.

Figure 1 represents total-to-total pressure coefficient, $\psi=\left(P_{2}-P_{l}\right) /\left(0.5 \rho U_{t}^{2}\right)$, and overall efficiency against the flow coefficient, $\phi=\bar{U} / U_{t}$, where $U_{t}$ denotes the blade tip velocity. Static and dynamic pressure coefficients are also included in the figure. The overall efficiency relates the power transferred into the air stream and the power supply of the driving motor. The three operating points that were studied, defined as "nominal" (Qn), "partial load" (0.85Qn) and "near stall" $(0.7 \mathrm{Qn})$, are shown in the figure. The nominal point corresponds to the peak efficiency of the fan performance, at $\phi=0.38$. The uncertainty of the curves has been estimated in $\pm 2 \%$ for the flow rate and $\pm 1.4 \%$ for the total pressure. With this level of accuracy, no relevant differences have been observed in the performance curves for both gap configurations.
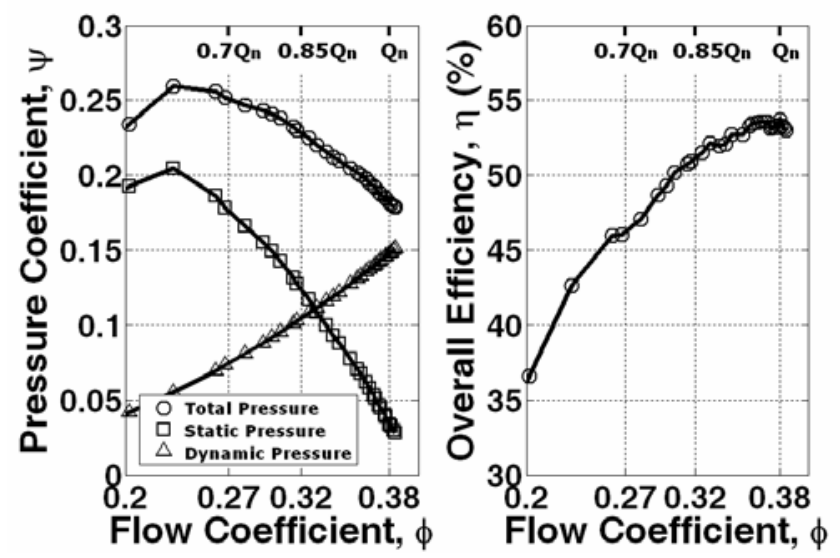

Fig. 1. Fan performance curves. 
Table 1. Characteristics of vanes and blades.

\begin{tabular}{|c|c|c|c|c|c|c|}
\hline \multirow[t]{2}{*}{ Radius (mm) } & \multicolumn{2}{|c|}{$\begin{array}{c}\text { HUB } \\
190\end{array}$} & \multicolumn{2}{|c|}{$\begin{array}{c}\text { MIDSPAN } \\
300\end{array}$} & \multicolumn{2}{|c|}{$\begin{array}{l}\text { TIP } \\
410\end{array}$} \\
\hline & Stator & Rotor & Stator & Rotor & Stator & Rotor \\
\hline Chord length (mm) & 157.0 & 179.0 & 165.3 & 165.8 & 170.8 & 157.4 \\
\hline Solidity & 1.71 & 1.35 & 1.14 & 0.792 & 0.86 & 0.55 \\
\hline Stagger angle $\left({ }^{\circ}\right)$ & 18.11 & 48.64 & 12.97 & 59.70 & 10.77 & 66.45 \\
\hline Camber angle $\left({ }^{\circ}\right)$ & 37.1 & 13.76 & 27.8 & 7.42 & 23.3 & 5.74 \\
\hline Thickness-to-chord ratio (\%) & 3.0 & 12 & 3.0 & 9.43 & 3.0 & 8 \\
\hline Pitch (mm) & 91.8 & 132.6 & 145 & 209.4 & 198.1 & 286.2 \\
\hline Inlet flow angle $\left({ }^{\circ}\right)$ & 0.0 & 59.61 & 0.0 & 64.7 & 0.0 & 69.36 \\
\hline Outlet flow angle $\left({ }^{\circ}\right)$ & 31.17 & 47.73 & 20.73 & 60.07 & 15.68 & 67.16 \\
\hline
\end{tabular}

Measurement techniques. Dual hot-wire anemometry. Hot-wire techniques were used in order to measure the velocity field across the stage. This technique gives a relatively high frequency response to velocity fluctuations, so flow disturbances at BPF (360 $\mathrm{Hz}$ ) can be perfectly captured. The anemometric probe was composed of two tungsten filament wires of $5 \mu \mathrm{m}$ dia, in a $120^{\circ}$ crossed layout, with output signals connected to a TSI IFA100 constant temperature anemometer. The frequency response of the wires was estimated to be about 20 and $30 \mathrm{kHz}$, respectively. The angular uncertainty has been estimated at $1^{\circ}$ in the center of the measurement range and $3^{\circ}$ at the borders. The uncertainty of the absolute velocity value has been estimated at $1.3 \%$ in the center and up to $3 \%$ near the borders. More details about the probe and the calibration setup can be found in Blanco et al, [12].

Figure 2 shows the measurement locations used in the experiments. Station A, placed far upstream of the stator, consists of 15 radial points covering the span of the machine. Next, Sector D was placed $15 \mathrm{~mm}$ behind the stator TE in order to capture flow patterns between the rows. Finally, the Sector R was located $50 \mathrm{~mm}$ downstream of the rotor TE in order to analyze the rotor wake velocity deficit. Since there are no clocking effects of additional stages, all the periodic unsteadiness is observable inside a circular sector covering the whole span of the machine over one stator pitch. As in Station A, 15 single points were adopted over the span in both sectors. On the other hand, another 15 angular positions -every $1.8^{\circ}$ - were used to complete the vane's passage definition in the tangential direction. Therefore, two "windows" of 225 measuring points were finally defined in both sectors D and R (figure 3 ).

To avoid aliasing of the signals, the data sampling rate was fixed at $36 \mathrm{kHz}$, that is, a temporal resolution of 100 samples per blade passing period. For every velocity trace, 100 rotor blade passing periods were recorded approximately, so a total number of $10^{4}$ samples were stored at each measuring point of the $15 \times 15$ sector grid. A continuously recording trigger, which is fixed to the rotor, enables a correct identification of every rotor phase. Thus, accurate data sets can be rearranged from every velocity trace in order to phase-average the instantaneous signals.

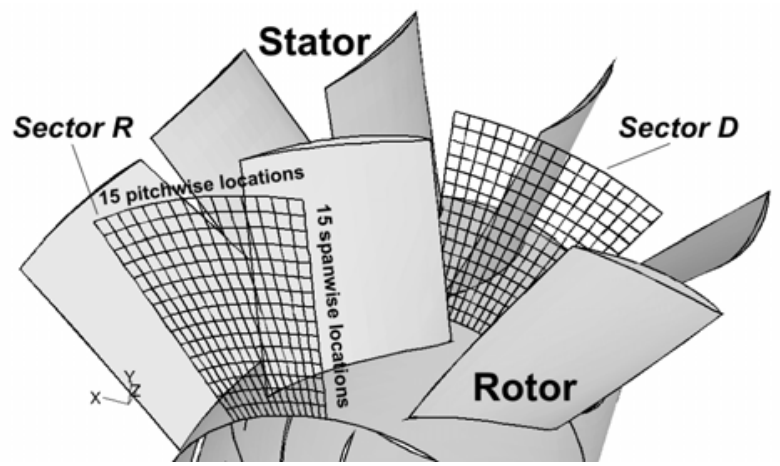

Fig. 3. Measuring sectors $D$ (at stator exit) and $R$ (at rotor exit).

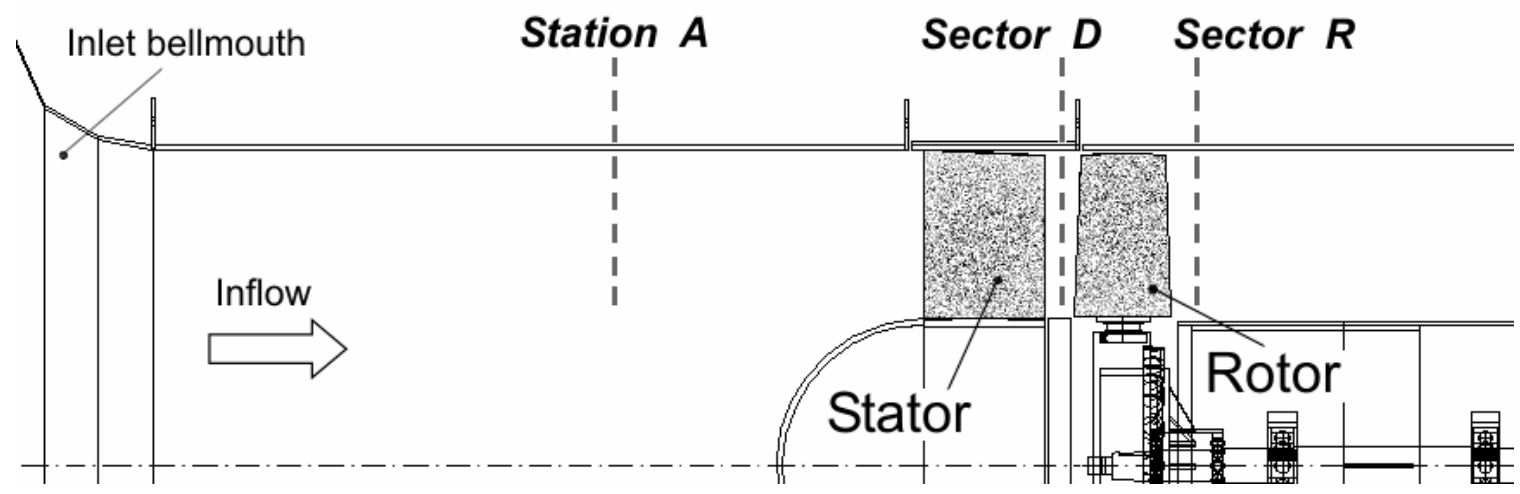

Fig. 2. Measurement locations. 


\section{Data processing techniques.}

Ensemble and Time-Averaging. The hot-wire traces have been processed in order to segregate periodic fluctuations from random unsteadiness. Since the velocity fluctuations are considered to have a random (turbulent) component and a deterministic component, the instantaneous velocity can be decomposed into a periodic, deterministic velocity and a stochastic function as follows:

$$
u(\vec{r}, \vartheta)=\tilde{U}(\vec{r}, \varphi)+u^{\prime}(\vec{r}, \vartheta)
$$

where the deterministic velocity is obtained by ensemble-averaging (or phase-averaging) the hundred passages recorded for every velocity trace $(M=100$ rotor blades periods stored). The ensemble-averaging is defined as:

$$
\tilde{U}(\vec{r}, \varphi)=\left.\frac{1}{M} \sum_{m=1}^{M} u(\vec{r}, \vartheta)\right|_{m} ; \vartheta=\varphi+\frac{2 \pi}{B}(m-1)
$$

The variable $\vec{r}$ represents the spatial coordinates and $\varphi$ is the phase angle of the rotor passage. $B$ is the number of blades and $\vartheta$ computes the total angular displacement. It has been defined a temporal resolution of 100 samples per blade passing period, so a single phase-locked position of the rotor pitch $\left(40^{\circ}\right)$ has been obtained every $0.4^{\circ}$. This phase-averaged flow leads to the final passage-averaged flow if the deterministic velocity is further decomposed into a mean value and a deterministic fluctuation.

$$
\tilde{U}(\vec{r}, \varphi)=\bar{U}(\vec{r})+U(\vec{r}, \varphi)
$$

Considering that the deterministic fluctuations occur on large time scales, they can be easily identified by timeaveraging (or passage-averaging) the deterministic velocity, according to:

$$
\bar{U}(\vec{r})=\left.\frac{1}{N} \sum_{n=1}^{N} \tilde{U}(\vec{r}, \varphi)\right|_{n}
$$

where $n$ represents every phase-averaged realization that covers an entire passage $(N=100)$. Notice that the phase $\varphi$ is removed in the time-averaged velocity $\bar{U}$, so passage-averaged variables are phase-rotor independent values (steady maps). Equation (5) gives the final decomposition of the instantaneous velocity in its more compact form, as the contribution of a mean value, $\bar{U}$, a deterministic fluctuation or "unsteadiness", $U$, and a pure chaotic randomness or "turbulence", $u$ '.

$$
u(\vec{r}, \vartheta)=\bar{U}(\vec{r})+U(\vec{r}, \varphi)+u^{\prime}(\vec{r}, \vartheta)
$$

FFT Filtering. In many cases, it may occur that the instantaneous velocity includes other "large-scale" unsteadiness that is periodic but not at the BPF. The existence of secondary flow patterns like sawing movement of the tip leakage vortex, fluttering of separated flows or unstable separation points ([6]) implies that the ensemble-averaging technique does not remove all the periodic components of the instantaneous velocity. As a result, the random component $u^{\prime}$ defined in (1) is not realistically describing pure turbulence, so an additional filtering method is needed to remove all the periodicity.

Following the methodology described in [4], a frequential analysis is employed to identify all the periodic events in the power spectrum of the instantaneous velocity signal. Thus, the peaks in the Fourier transform are digitally filtered out by setting its amplitudes to zero. The truncated power spectrum is then transformed back into the time domain giving the turbulent signal.

This procedure, justified in detail with a single velocity trace measured downstream of the rotor, will be discussed later on in the results section (figures 12 and 13).

Description of turbulence. Different variables can be used to describe the level and the spatial distribution of the turbulent flow. The turbulence intensity expresses the "strength" of the turbulent motion. It is defined as:

$$
T u(\vec{r}, \varphi)=\frac{\sqrt{\widetilde{u^{\prime 2}}}}{\tilde{U}}=\frac{1}{\tilde{U}} \sqrt{\left.\frac{1}{M} \sum_{m=1}^{M} u^{\prime 2}\right|_{m}}
$$

where the hat denotes the ensemble-averaging value of the random fluctuations to the square. In turbomachinery, the root mean square value (RMS) of the fluctuations is divided by the deterministic fluctuation, $\tilde{U}$, in order to obtain the unsteady turbulent field. This definition reveals the transport of the turbulent structures in the unsteady, deterministic flow patterns. In the case of there being no deterministic fluctuations, the ensemble-averaging would be replaced by a time-averaging.

The integral length scale gives an idea of the spatial dimension of the largest eddy size of the turbulent structure. As described in [1] and [4], if all the periodic events are removed from a velocity trace, then the autocorrelation function will show no oscillations, so the integral length scale, $L$, can be estimated by this expression:

$$
L=\bar{U} \int_{0}^{\infty} A C F(\tau) d \tau ; A C F(\tau)=\frac{\overline{u^{\prime}(t) u^{\prime}(t+\tau)}}{\overline{u^{\prime 2}}}
$$

where the overbar denotes the time-averaging value, and $\tau$ is the time lag that is used to construct the ACF. This formulation supposes the validity of Taylor's hypothesis (the average eddy size lies through the correlation of two velocity signals). Obviously, the time lag depends directly on the data sampling rate, so an average eddy of a wave number larger than the sampling rate cannot be measured. 
At a sufficiently high Reynolds number, in the case of fully developed, nearly isotropic turbulence, the spectrum of turbulence exhibits an inertial subrange, characterized only by the energy dissipation rate, $\varepsilon$. Dimensional analysis allows to derive an analytical form of the spectrum: $E(k)=\alpha \varepsilon^{2 / 3} k^{-5 / 3}$, where $k$ represents the eddies wavenumber. In the literature, much experimental data confirms the existence of this power-law region, with $\alpha=1.5$ as a typical value of the Kolmogorov's constant. In this inertial subrange, an energy cascade is established and the turbulent energy is derived from larger eddies and gradually transferred to smaller eddies until it is dissipated. Therefore, the viscous dissipation of energy can be estimated from the large-scale dynamics according to $\varepsilon \sim \frac{u_{L}^{3}}{L}$, where $u_{L}$ is a characteristic velocity scale of the largest eddies. It can be perfectly assumed that this velocity scale has the same order of the random velocity fluctuations (Tennekes et al., [13]). As a consequence, the powerlaw spectrum can be estimated for every velocity trace, using the RMS value of the chaotic unsteadiness in the definition of the energy dissipation rate.

\section{EXPERIMENTAL RESULTS AND DISCUSSION}

Inflow turbulence. Figure 4 shows the turbulence level of the streamwise velocity along the radial coordinate at the inlet. Since Station A is placed far upstream from the rotor, there are no periodic events involved in the velocity traces. As a consequence, both ensembleaveraging and FFT filtering are not necessary in this case. The plot compares the three different operating points (nominal -Qn-, partial load -0.85Qn- and near stall -0.7Qn-) for the larger axial gap configuration. There are no significant variations in the level of turbulence with the mass flow rate because Re is high enough (in the range of $1.2 \times 10^{6}$ ) to make turbulence statistically independent of low $\mathrm{Re}$ changes. As expected, the results show a clear spanwise uniformity, except for the casing viscous region where higher levels of turbulence (up to $9 \%$ ) are produced. A typical value of $1.5 \%$ is obtained for the free-stream region, which is totally representative for this type of open-loop facilities.

Figure 5 shows a radial distribution on the integral length scale at the fan inlet. Due to the inherent randomness of these fluctuations, there is an appreciable radial disparity in the results. However, for this Reynolds range, the characteristic scale of larger eddies turns to be in the order of one hundred and thirty milimeters, with a clear spanwise uniformity. As expected, these larger eddies have velocity and length scales comparable to the global scales of the flow, because the integral value is a fraction of the duct diameter (approx. 16\% of $820 \mathrm{~mm}$ ). Complementarily, the figure includes the autocorrelation function of one radial point, needed to estimate the integral scale. To evaluate the area under the ACF, the first zero in the autocorrelation is usually taken as the upper limit of integration in (7) [1, 4].

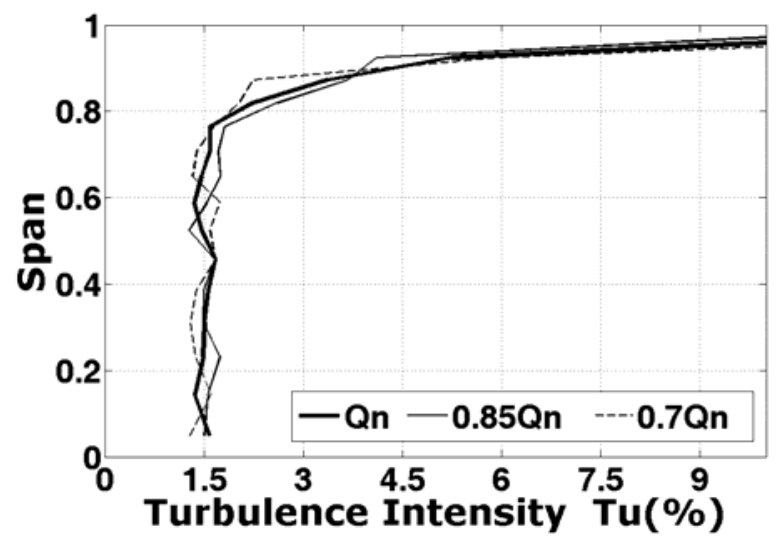

Fig. 4. Turbulence level of streamwise velocity of the inlet flow.

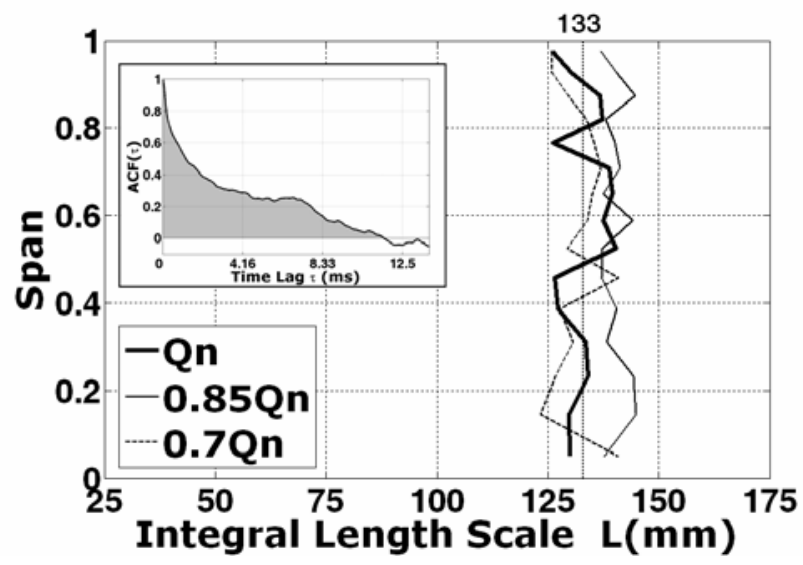

Fig. 5. Radial distribution of the integral length scale of the inlet flow.

The idea of the integral length scale as a characteristic value of the larger eddies of the flow is revisited in figure 6 . This figure shows a short fragment of the random fluctuations obtained from a particular velocity trace at Station A. The amplitude of the fluctuations, about $0.5 \mathrm{~m} / \mathrm{s}$, represents a $1.6 \%$ of the mean velocity (in agreement with the average turbulence level of figure 4). Considering that the measuring probe is fixed at one particular position, the temporal signal is really showing the structure of the flow passing by. Thus, when a small eddy transported by the mean flow reaches the probe, it leaves an abrupt, sharp fingertip in the trace, since it takes a short time to pass through. On the contrary, the contribution of the largest eddies is more like a large, background fluctuation, because they take a long time to pass by. This is sketched in the figure, where large eddies are identified with repetitive background oscillations. Therefore, the average size of large eddies can be estimated noticing that they take about $4 \mathrm{~ms}$ (see the figure) to cross the probe when they are transported at a mean flow velocity of $31 \mathrm{~m} / \mathrm{s}$. Then, this estimation of the characteristic length scale, approx. $124 \mathrm{~mm}$, is in perfect agreement with the order of magnitude in figure 5!. Obviously, this one-dimensional description is a quite simplistic approach to the complex picture of 
turbulence, but it gives a very useful idea of the turbulent scales involved in the flow.

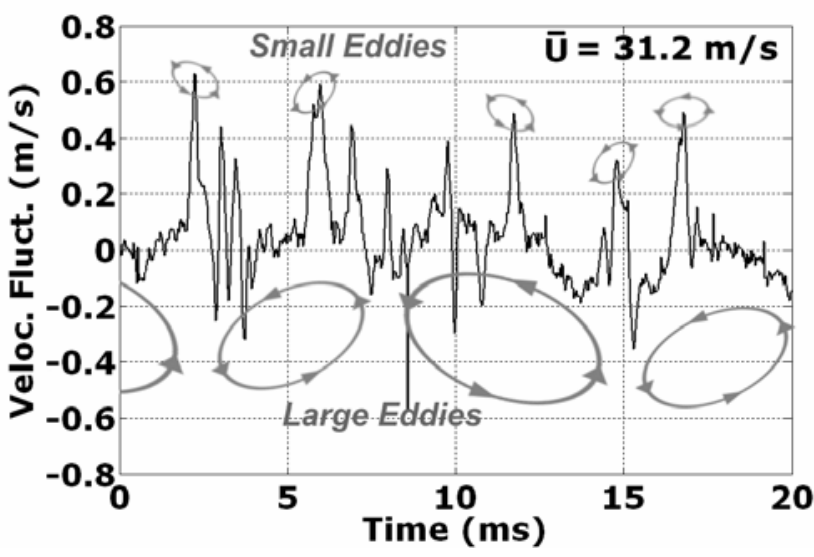

Fig. 6. Simplistic sketch of the contribution of large and small eddies in the random fluctuations of the velocity traces for fully-developed turbulence. (Adapted from [14]).

Finally, figure 7 compares the power spectrum of velocity fluctuations between free-stream (black) and casing viscous (grey) regions at Station A. Both frequency and power axis are plotted on a logarithmic scale, where the fluctuations were made nondimensional by the mean-time velocity. Outside the viscous boundary layer, the turbulence exhibits a nearly isotropic, fully-developed decay, following the universal power-law in the inertial subrange. The dissipation rate has been calculated using the average integral length scale shown in figure 5 and the level of turbulence of figure 4. On the contrary, within the casing viscous region, the power spectrum shows higher levels throughout the frequency range. Because of the high small-scale turbulence generated in the boundary layer, small turbulent eddies shed from the shear layer start to redistribute their energy over a broader range of eddy sizes. This means that the energy spectrum broadens, searching the fully-developed state, so the slope of the inertial subrange must be greater than the $-5 / 3$ exponential decay (Davidson, [14]). Similar results have been encountered by Goto ([6]) in axial flow compressors.

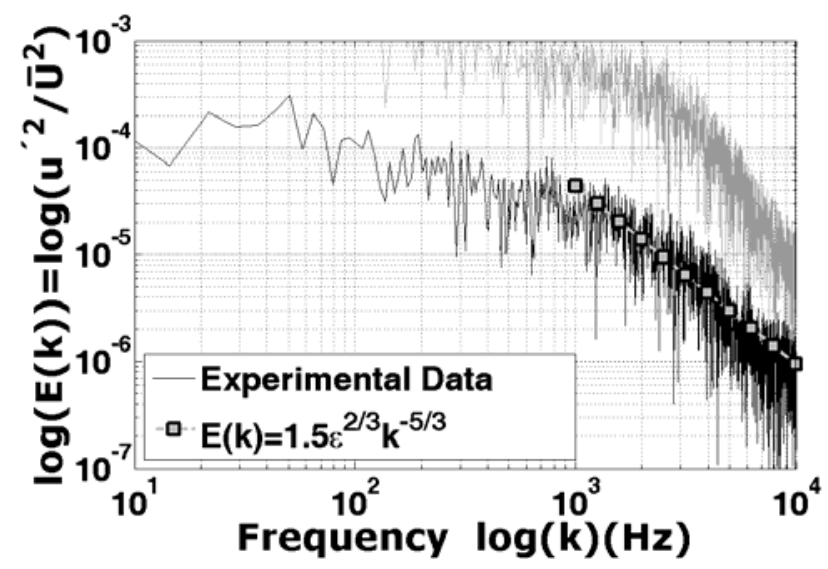

Fig. 7. Power spectrum of velocity fluctuations at free-stream and viscous regions in Station $A$.
Unsteadiness and Turbulence at Stator Exit. The unsteadiness at the stator exit is mainly generated by the potential effect of the rotating blade surfaces, which are felt like a periodic blockage on the phase-averaged flow (Fernández Oro, [15]). The analysis of this inviscid mechanism requires an ensemble-averaging technique in order to decouple this deterministic fluctuation from the total unsteadiness. On the other hand, there is no need of FFT filtering on Sector D because no vortical disturbances of the rotor are propagated upstream. This is noticeable from the FFT spectra of instantaneous velocity (not shown here) where it can be observed that there is no contribution of high-order periodicity.

Figure 8 shows turbulence intensity levels between the rows for both larger and lower axial gap configurations at nominal conditions. These results, computed from (6), correspond to the midspan position. They have been replotted over a second stator passage in the horizontal axis, since the flow is periodic in the pitchwise direction. The vertical axis shows time nondimensionalized by the rotor blade passing period $\left(t / T_{r}\right)$. These temporal maps are plotted using a scale ranging from a minimum value of $2.25 \%$ to a maximum peak of turbulence intensity of $12 \%$ within the stator wakes. Turbulent stator wakes are clearly defined by vertical bands of high turbulence at a particular tangential position in the plots. At the larger axial gap (figure 8, top), there is no influence of the rotor blockage on the turbulence levels in the stator passage. Only minor variations over time are observable in the core of the stator wakes, indicating an interaction of the blades stagnation conditions with the wakes turbulent structure. When the lower axial gap situation is considered (figure 8, bottom), the lower threshold in the figure exhibits an appreciable increase in the turbulence level because of the unsteady blockage of the rotor blades in the stator passage (see grey zones). Thus, additional turbulence associated with this blockage is observed running diagonally across the map. The slope of these turbulent structures derives from the relative number of vanes and blades. It means that during every blade passing period these turbulent structures are transported along $13 / 9$ of the stator pitch by the unsteady flow patterns. In addition, it must be pointed out that the turbulence level within the stator wakes is also influenced by the rotor blockage. In this case, the rotor blockage decreases the turbulence intensity in the stator wakes, thus inducing a calm region as the blades are passing by.

In figure 9, the line graphs show the time evolution of velocity fluctuations and turbulence levels at midspan for two angular positions. Both larger and lower axial gaps have been represented again at nominal conditions. In particular, the black lines correspond to a tangential location influenced by stator wakes, say at a stator pitch value of 0.5 in figure 8 ; whereas the grey lines lay with the stator midpassage, say at a pitch value of 1 . 

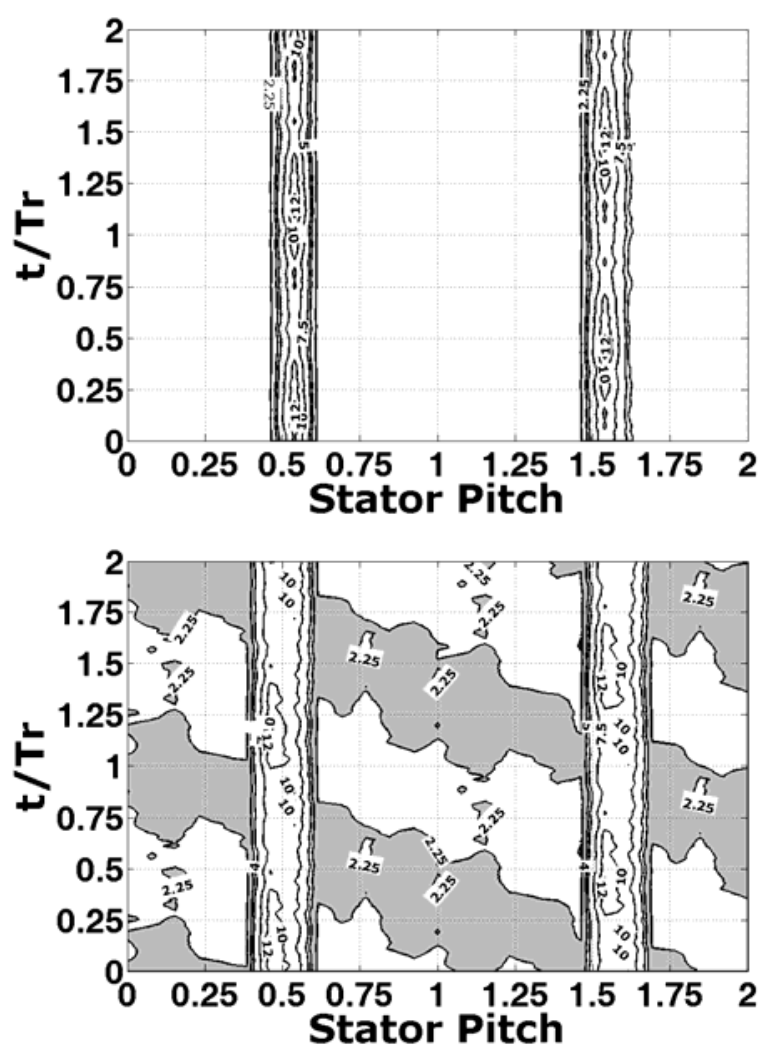

Fig. 8. Turbulence intensity level at midspan in case of nominal conditions for both larger (top) and lower (bottom) axial gaps.

Both instantaneous $(u / \bar{U}$, in dashed lines $)$ and ensemble-averaged $(\tilde{U} / \bar{U}$, in solid lines) velocities are clearly showing the rotor blockage on the unsteady flow structures. Notice that this effect is more pronounced on the stator wakes than in the midpassage due to a major small-scale turbulence induced on the wakes shear layers. In fact, there are very small differences between both instantaneous and averaged velocities at midpassage, indicating a high-stable, potential behavior of the flow on that zones. Meanwhile, the small-scale turbulence induced by the rotor blockage is increased as the axial gap is reduced because of the major enhancement of the vortical instabilities at the shear layers. In this case, even the influence of both pressure and suction sides of the rotor blades is identifiable in the ensemble-averaged velocity traces within the stator wakes.

In addition, turbulence levels are also represented in both wake and midpassage positions for the two axial gap configurations. Here what is remarkable is the opposite trend shown by both positions as a function of the axial gap. Thus, in the larger axial gap case, the rotor blockage is observable in the stator wake structure, whereas in the lower gap configuration the rotor blockage is present only at midpassage. On the other hand, this inviscid mechanism is lost in the wake structure of the lower axial gap configuration due to the high small-scale unsteadiness that engulfs the large-
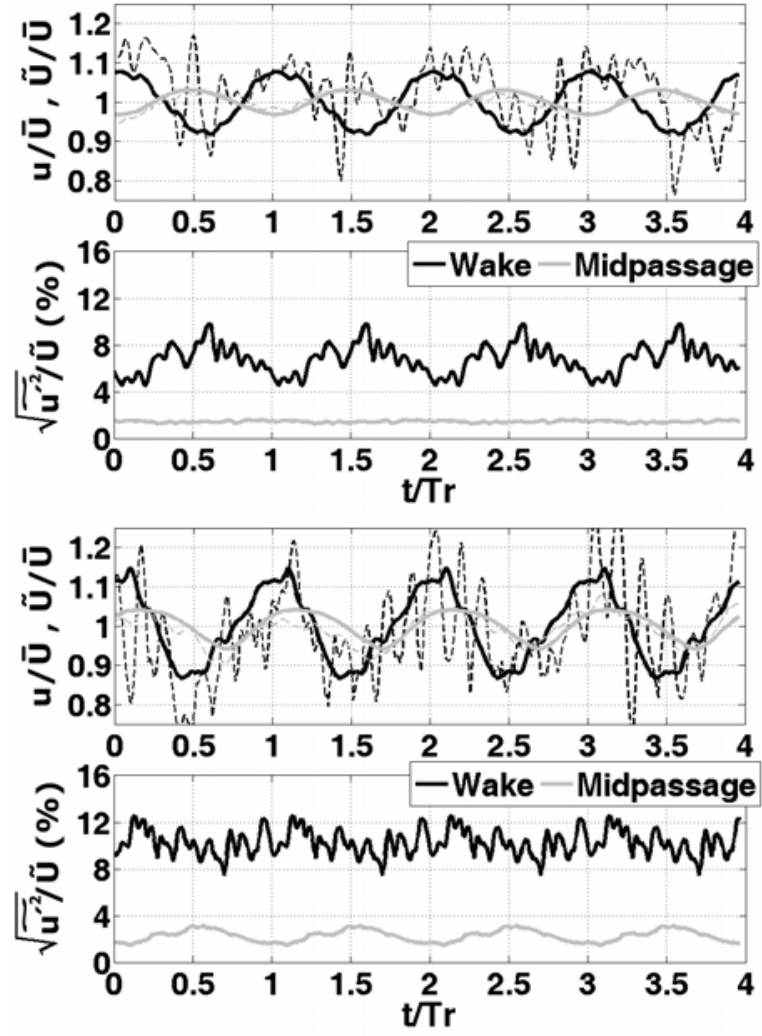

Fig. 9. Time evolution of instantaneous and ensembleaveraged velocities as well as turbulence levels at midspan in case of nominal conditions. Both larger (top) and lower (bottom) axial gaps have been represented for two significative pitchwise positions.

scale fluctuation of the blockage (previously discussed in the velocity traces). Time-averaged level of turbulence within the wakes is estimated in a $10 \%$, with a background turbulence of approximately $2 \%$ at midpassage (increased from the inlet flow level), in case of the lower axial gap. Similar values were found by Senkter et al., [7] in low-speed axial compressors. These values are reduced to $6 \%$ and $1.5 \%$ respectively in the case of larger axial gap.

The influence of the operating point is outlined in figure 10 , which represents the same variables at the same locations of figure 9 , but now in the case of near stall conditions (0.7Qn). Basically, similar conclusions to those in figure 9 can be derived when the flow rate is reduced. Only an increase on the overall turbulence levels is noticeable because of a higher total disturbance level at off-design conditions (see also Velarde-Suárez et al., [10]). Thus, turbulent intensities increase to $12 \%$ within the wakes and 3\% of background turbulence in the case of lower axial gap; and $8 \%$ and $2.5 \%$ respectively for the larger axial gap.

Integral length scales at the stator exit are shown in figure 11 for both nominal (Qn) and near stall (0.7Qn) operating conditions. The maps indicate that the largest length scales are encountered in the stator midpassage (in the order of $80 \mathrm{~mm}$, that is, a $50 \%$ of the blade chord), showing high averaged values as a result of the convection of larger eddies developed far upstream. 

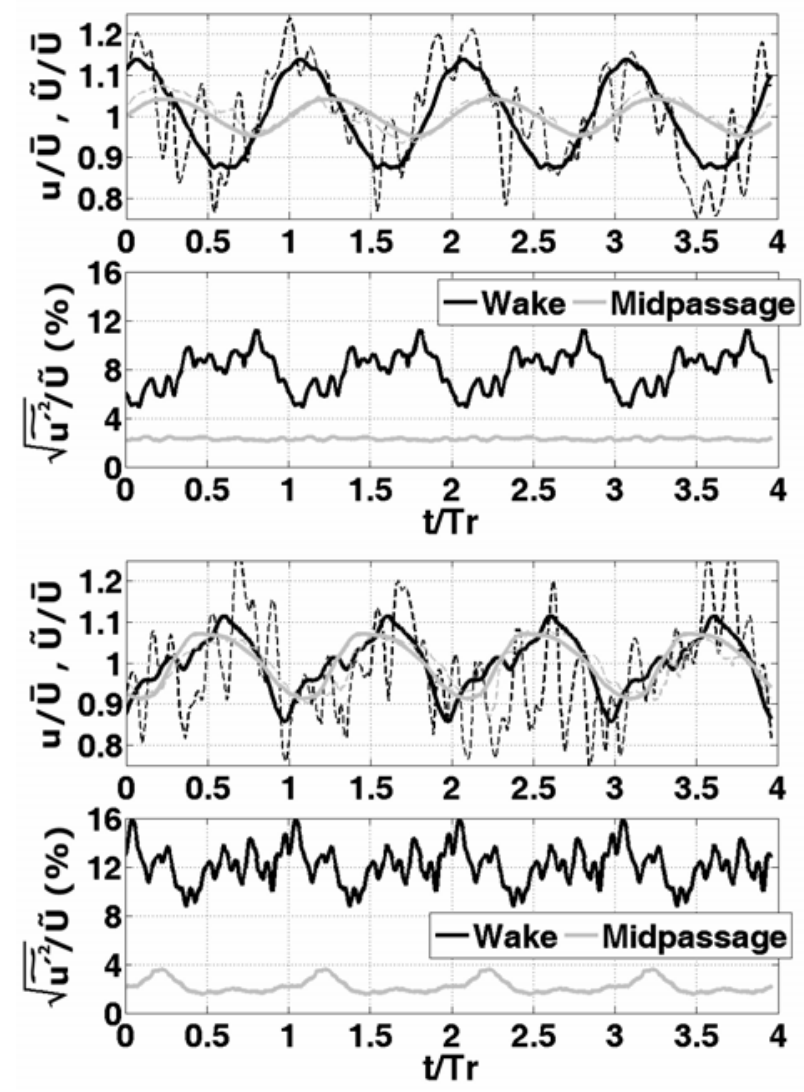

Fig. 10. Time evolution of instantaneous and ensemble-averaged velocities and turbulence levels at midspan in the case of near stall conditions. Both larger (top) and lower (bottom) axial gaps have been represented for two particular pitchwise positions.

As these large eddies are transported throughout the stator, they are chopped by the stator vanes, resulting in characteristic sizes in the range of the stator pitch (see table 1). On the other hand, the casing boundary layer and the stator wakes are important sources of turbulence contributing with a very low integral length scale. Therefore, instabilities and vortices shed from these shear layers present integral length scales in the order of one millimeter (a fraction of the blade chord). In this case, turbulence from the stator wakes has an integral length scale of approximately $5 \mathrm{mms}$ ( $3 \%$ of the vane chord), in reasonable agreement with previous predictions by Camp et al., $2 \%$, or Henderson et al, $5.4 \%$, in 4-stage and 1.5-stage axial compressors, respectively. In the case of a lower flow coefficient, the casing boundary layer is increased becoming the predominant mechanism in the establishment of the integral length scale distribution. Thus, the overall length scale is reduced with smaller eddies constituting the essence of the turbulent structure.

Unsteadiness and Turbulence at Rotor Exit. The transport of the velocity deficit of rotor wakes is the basic mechanism that produces an unsteady scenario inside multistage turbomachinery. Since several wake streets are established according to the number of blades, velocity variations due to a lattice of wakes can be described as periodic flow fluctuations. At this point it must be noticed that, although the velocity deficit exhibits a truly periodic feature in itself, the vortical nature of the shear layers confining the velocity deficit could introduce other large-scale phenomena with highorder periodicity. Therefore, the segregation of periodic and non-periodic components of the flow could be compromised when using an ensemble-averaging technique at the BPF. Thus, for all the results at the rotor exit, the FFT Filtering has been introduced to guarantee an accurate decoupling method.
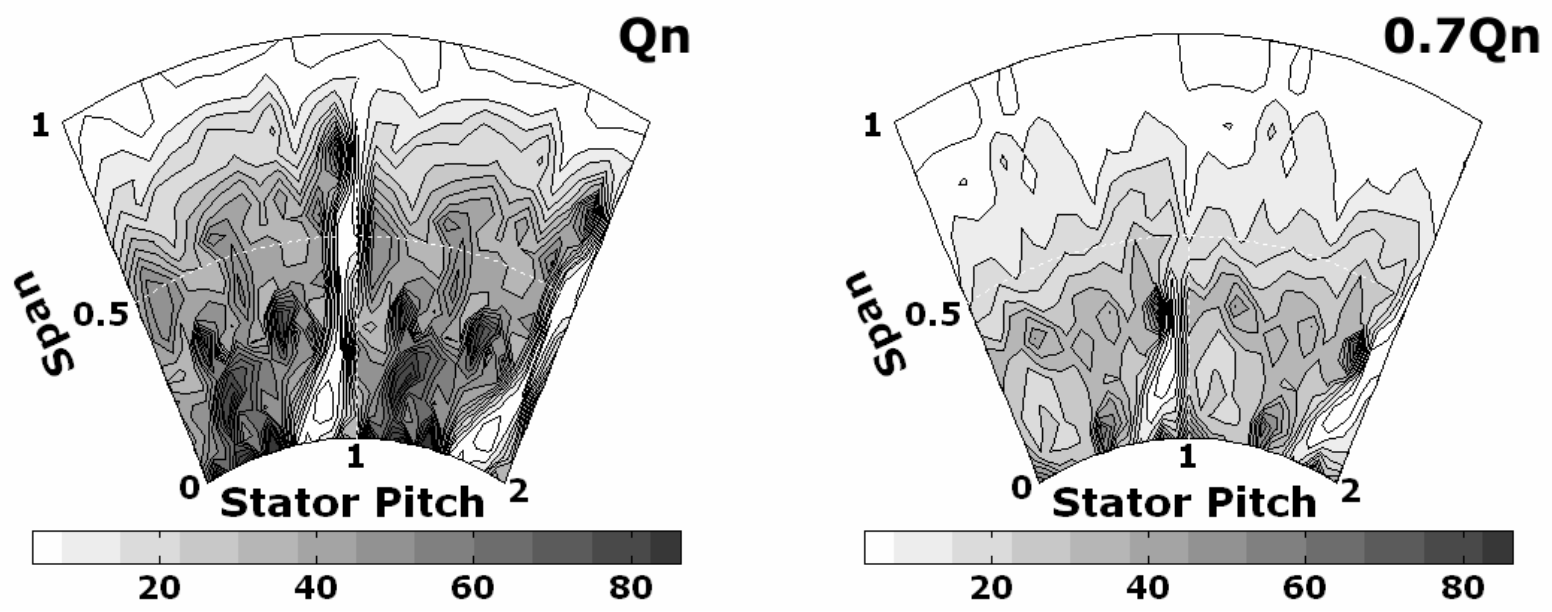

Fig. 11. Distribution of integral length scales (in $\mathrm{mm}$ ) at the stator exit for both nominal and near stall conditions. 
Figure 12 shows power spectra of both periodic and random components of a velocity trace measured downstream of the rotor. In the subplot 12.a, peaks corresponding to the BPF and its harmonics are clearly visible in the spectrum of the instantaneous velocity. Also, the contribution of periodic unsteadiness related to the machine rotational frequency is observable in the spectrum. Next, in subplot 12.b the power spectrum of the ensemble-averaged velocity shows a perfect periodic nature with the BPF. As a result, there is no contribution to large-scales below $360 \mathrm{~Hz}$, while higher harmonics appear to configure the particular shape of the blade-toblade periodic velocity. Subtracting the ensembleaveraged velocity to the instantaneous signal, the pure fluctuation is expected to be obtained in subplot 12.c. Nevertheless, the power spectrum does not show the characteristic broadband distribution along the whole range of frequencies that should appear in the case of pure randomness. In particular, high-order periodicity is not totally removed by the ensemble-averaging technique. Thus, from the second harmonic on, there is still a very important reminiscence of periodic unsteadiness at BPF harmonics, though the fundamental peak of BPF has been filtered out in the procedure. Furthermore, even the rotational frequency periodicity is present in the fluctuation because the ensembleaveraging definition cannot distinguish between rotor revolutions. This observation underlines that temporal averaging alone is not sufficient to eliminate all the periodicities. The power spectrum, $E(k)$, is the distribution of energy across the different eddy sizes, but every eddy size is really contributing across the full range of wave numbers ([14]). In addition, periodic disturbances are superimposed to that sea of all-range eddies in a similar fashion. Thus, the combination of both energy densities derives in a high complex structure with no exact frontier separating the periodic unsteadiness of large-scale fluctuations from the largescale eddies of random turbulence. In other words, the ensemble-averaging is considering that such a frontier can be established at the BPF wavenumber, and as a consequence, residual energy of large-scale fluctuations (or high-order periodicities) is masked as large-scale turbulence.

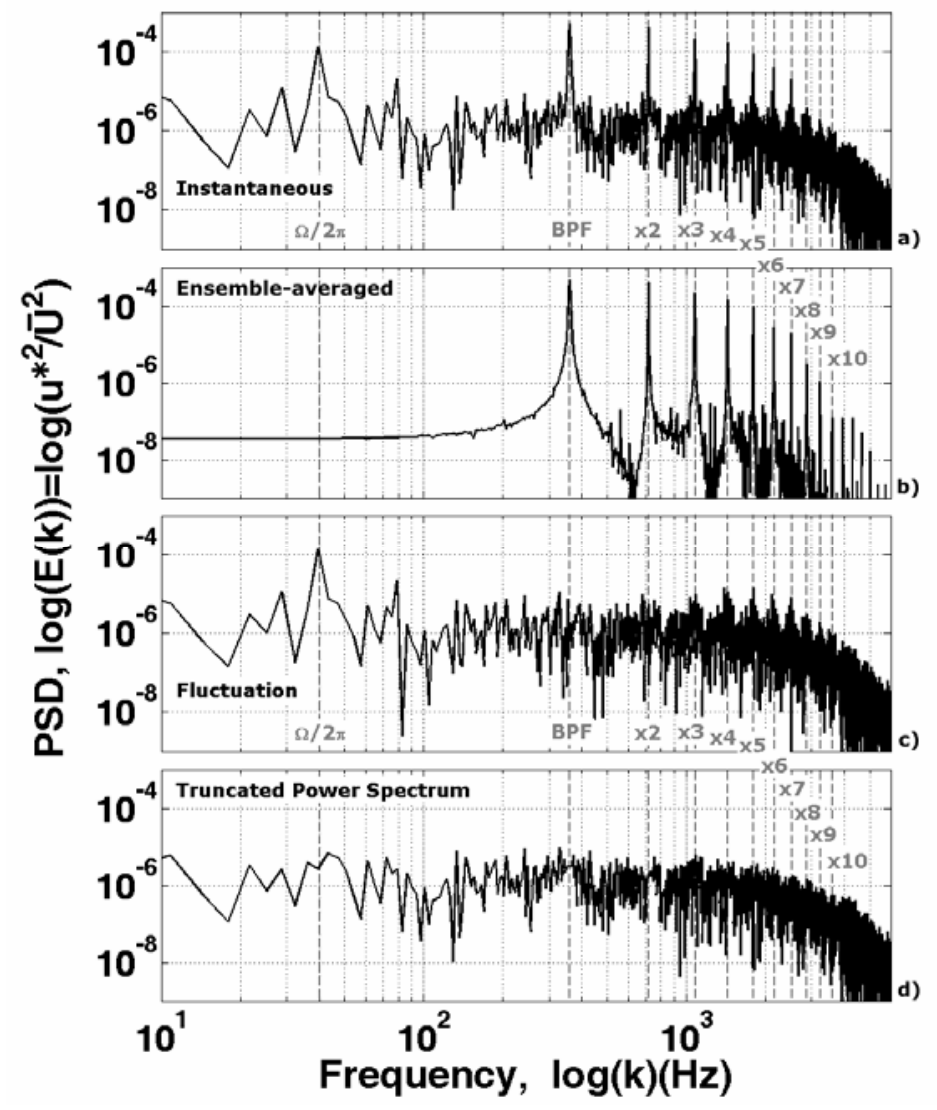

Fig. 12. Power spectra of the velocity components defined through (1) to (5) of an individual trace measured downstream of the rotor. $u^{*}$ corresponds to different definitions according to: a) Instantaneous: $u^{*}=u$; b) Ensemble-averaged: $u^{*}=\tilde{U}$;

c) Fluctuation: $u^{*}=u^{\prime}$; d) Truncated Power Spectrum,

$$
u^{*}=\left\langle u^{\prime}>\right.\text {. }
$$
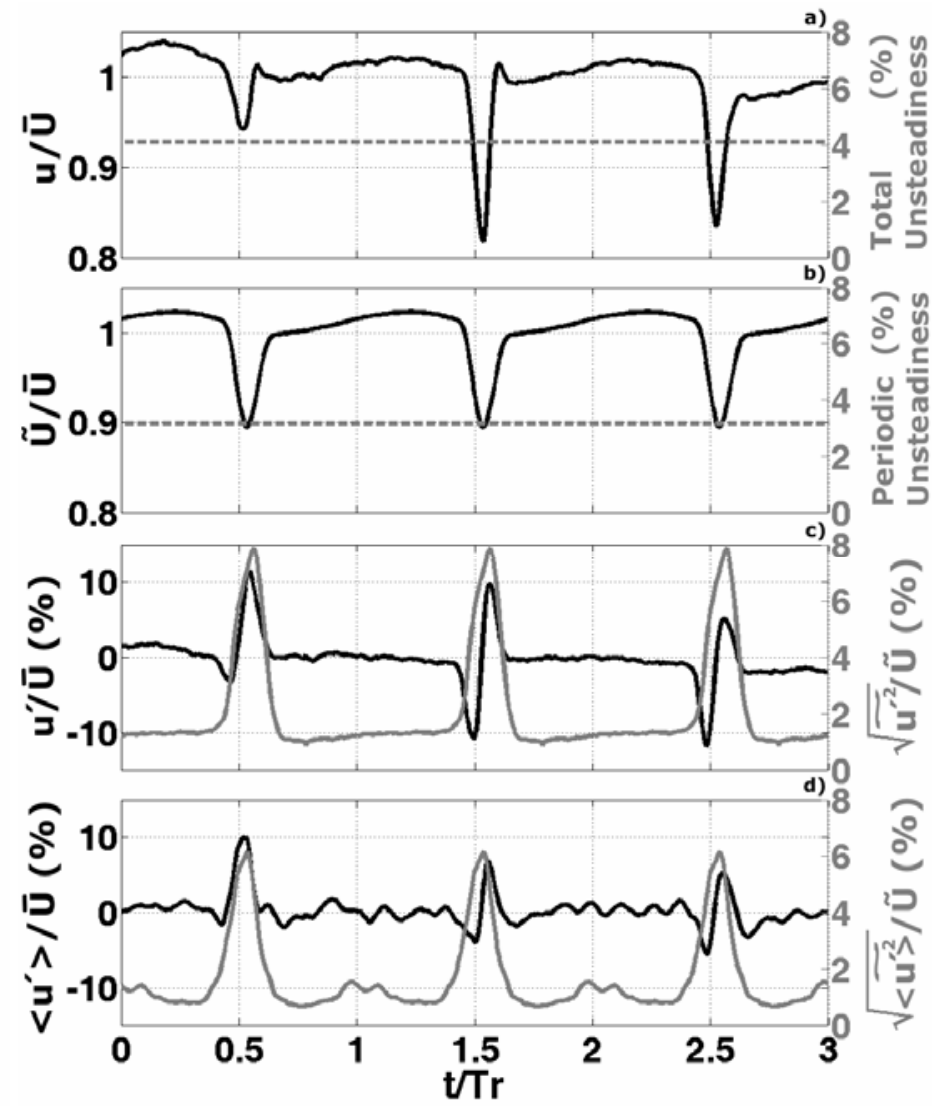

Fig. 13. Time evolution of the velocity components and turbulence levels defined through (1) to (6) for an individual trace measured downstream of the rotor. a) Instantaneous velocity $(u / \bar{U})$. b) Ensemble-averaged ( $\tilde{U} / \bar{U})$. c) Fluctuation $\left(u^{\prime} / \bar{U}\right)$. d) Filtered fluctuation $\left(<u^{\prime}>/ \bar{U}\right)$. 
Finally, the subplot 12.d shows the truncated power spectrum of the instantaneous velocity trace after FFT filtering. Using an iterative procedure, all the periodicity is removed from the instantaneous signal and a more realistic description of the turbulence is achieved. In fact, both fluctuation (12.c) and truncated spectra (12.d) contain the same small-scale, turbulent budget, despite being derived from different procedures (see the broadband component of both spectra). Thus, the usefulness of the truncated spectrum is shown because only chaotic randomness remains in the FFT signal, and all strong periodic events (marked with grey dashed lines in the plots) are completely filtered out.

The time evolution of the velocity components is used in figure 13 to illustrate the segregation process that leads to the description of pure turbulence. The same measuring point of previous power spectra is represented for that purpose. Complementarily, levels of unsteadiness and turbulence intensities are also defined to provide an order of magnitude for the different variables. Then, subplot 13.a shows the instantaneous velocity trace, nondimensionalized by the mean-time value as usual, over three rotor blade passing periods. The velocity deficit does not show an exact repeating pattern in every wake due to the inherent degree of randomness within the velocity trace; besides, a level of total unsteadiness can be defined by computing the RMS value of the wakes total fluctuation. In this particular trace, it gives a reference level of approximately a $4 \%$. Introducing a statistical approach by means of ensemble-averaging, all the velocity deficits are reduced to a single blade-to-blade deterministic fluctuation, which is shown in subplot 13.b (replotted three times). All the randomness has been filtered out, and total variations are therefore reduced. As a consequence, the level of unsteadiness of this statistic pattern decreases: approximately, at 3.5\%. Afterwards, the temporal subtraction of previous traces provides a quasi-random fluctuation (including highorder periodicities) in subplot 13.c. Obviously, there is a background level between the wakes (centered in zero) and large variations (up to a $10 \%$ ) only on the velocity deficits. According to (7), an unsteady evolution of the turbulence level can be defined in order to reveal how turbulence is transported by the unsteady deterministic flow. As a consequence, only in the wakes, there is a sudden increase in the turbulence level (reaching 8\%), whereas between the wakes, a background level of roughly $1.5 \%$ is established for calm regions. Unfortunately, results derived from 13.c are not strictly accurate. Figure 12 has justified how they may have been overestimated, because of the contribution of highorder periodicities masked as large-scale turbulence in the shear layers of the wakes. To overcome this problem, the filtering technique used in the present work, provides a more realistic random fluctuation, shown now in subplot 13.d. This real fluctuation exhibits more random features, even in the calm regions between the wakes. Since high-order periodicities included in the wakes shear layers are removed, the random fluctuation within the wakes is reduced. As a direct consequence, just $6 \%$ of random fluctuation is measured in the rotor wakes, and the background level is also reduced to approximately $1 \%$.

Figure 14 gives a very precise picture of the transport of turbulence structures across the stage. Furthermore, the interaction between rotor and stator wakes and the influence of the mass flow rate conditions on the global disturbance level is also highlighted in the following considerations. This kind of map, already introduced in previous figure 8, shows temporal distributions of turbulence intensity along the stator pitch, at one radial position. In this case, the midspan is considered for nominal (top), partial load (middle) and near stall (bottom) conditions in the case of larger axial gap. Transversal bands of high turbulence level, crossing the plots diagonally, represent the rotor wakes. Obviously, they are diagonal because they are moving tangentially over time. The width of the rotor wakes is increased as the flow rate decreases, while their level of turbulence is elevated gradually. In addition, vertical bands of low turbulence level confirm the presence of unmixed stator wakes rotor downstream. Notice that these stator wake segments, resulting from rotor blade chopping, are increasingly rotated as the rotor blade loading increases. Moreover, the dispersion of both stator and rotor wakes is clearly increased when the flow rate is reduced. This fact fits in with previous results showing total unsteadiness in similar axial fans ([10]) indicating a higher disturbance level at high loads. Now, the analysis of turbulent structures at the rotor exit reveals that major disturbances are derived from higher wake dispersion at low flow rates. The same trend has been observed by Henderson et al., [1], who also found that elevated free-stream turbulence accelerates the mixing-out of IGV wakes. It is plausible, from the near stall results in the figure, to think that a major disorder in the stator wakes also leads to an early mixing-out of rotor wakes (major diffusion).

Figure 15 shows the time evolution of velocity fluctuations and turbulence levels for two particular angular positions picked from figure 14. Black lines correspond to a location influenced by the unmixed stator wake, while grey lines give information of the midpassage structure. Both instantaneous $(u / \bar{U}$, in dashed lines) and ensemble-averaged $(\tilde{U} / \bar{U}$, in solid lines) velocities were plotted as usual. Blade-to-blade distributions of velocity present a typical jet-wake structure with high levels of turbulence in the blade wakes. The velocity deficit in the wake-affected position is lower than in the midpassage because of the lower mean-flow velocity within the stator wake. As a consequence, the rotor wake has a major influence (higher deficit) at midpassage. The reduction of mass flow rate influences the shape of the distributions (Fernández Oro et al., [16]), thickening the wakes and increasing the loading at the pressure side. Then, the blade-to-blade evolution of velocity lacks uniformity at off-design conditions and instantaneous traces become more fluctuating. 

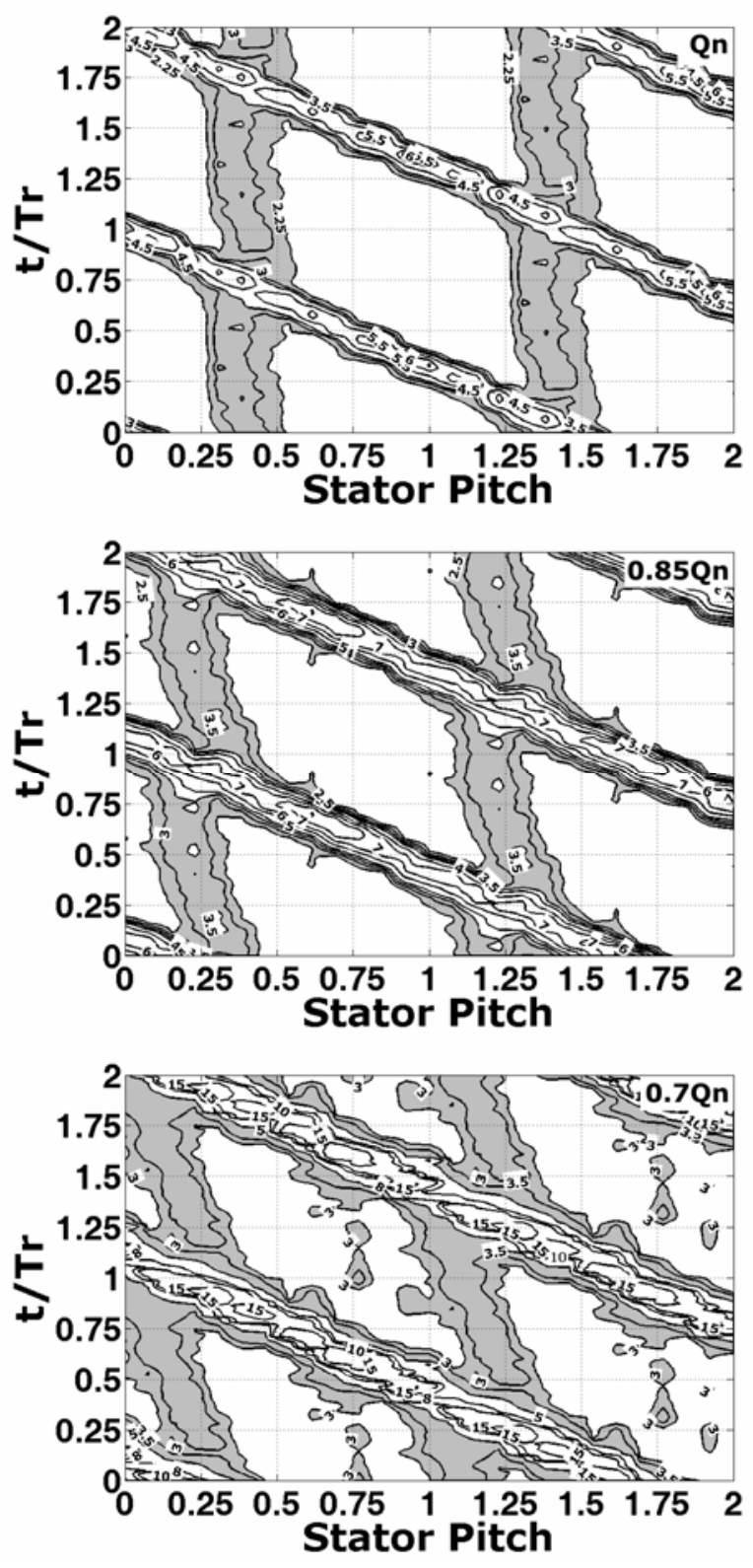

Fig. 14. Turbulence intensity level at midspan for nominal (top), partial load (middle) and near-stall (bottom) conditions in the case of larger axial gap.

On the other hand, turbulence distributions reveal three important characteristics of the turbulence structure downstream of the rotor. First, there is an overall increasing of the turbulence levels as the mass flow rate is reduced. Near stall conditions are especially significant because they present important background intensities (up to $5 \%$ ) with maximum peaks reaching $15 \%$ and even $20 \%$. Secondly, the maximum peaks are higher at midpassage locations than within the unmixed stator wakes. A similar trend was observed between the interaction of stator wakes and rotor blockage in figure 8. And thirdly, background levels of turbulence are much higher the in case of wake-affected positions than at midpassage. In this case, the high dispersion of the
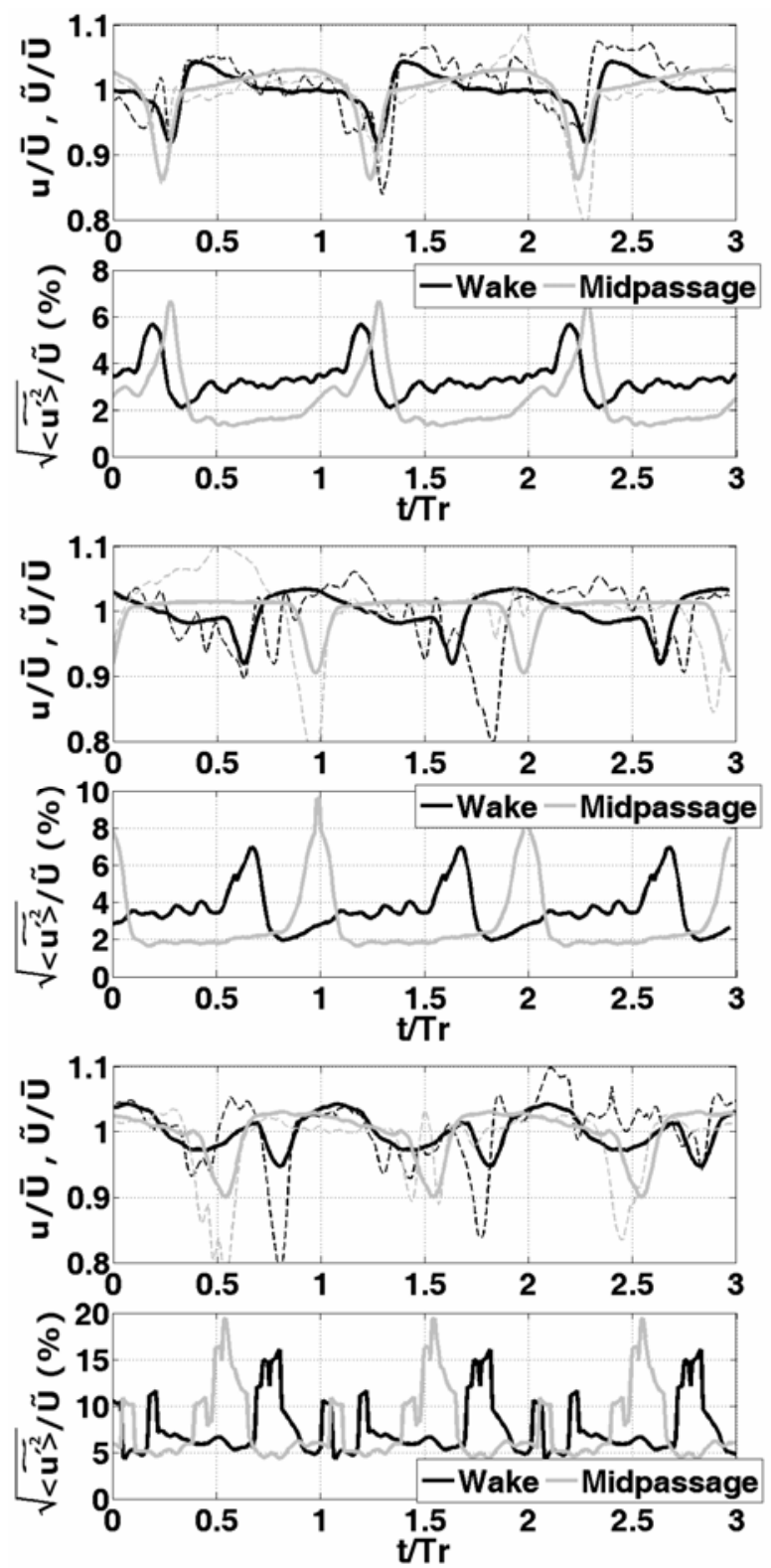

Fig. 15. Time evolution of instantaneous $(u / \bar{U})$ and ensemble-averaged $(\tilde{U} / \bar{U})$ velocities as well as turbulence levels at midspan for nominal, partial load and near stall conditions.

vortical disturbances shed from the stator wakes leads to high velocity fluctuations (compare instantaneous traces) and finally to a major generation of turbulence across the stage.

Final considerations of the turbulence structure downstream of the rotor are now illustrated in figure 16 . An instantaneous snapshot of the phase-averaged turbulence intensity is represented for both axial gap configurations at the three operating points tested in the experiments. Thus, the whole spatial distribution (radial and tangential) can be observed in Sector R. Figure 16.a (left column) shows results for the larger axial gap. The three operating points were reproduced in a similar instantaneous realization to facilitate the comparison. At 
nominal conditions (top), a snapshot view of a turbulent rotor wake is obtained at the relative stator pitch position of 1.2. This corresponds to a particular instant in time, in particular, $t / T_{r}=0.44$, according to an arbitrary convention of the time origin. Also, the stator wakes, fixed in time, are perfectly observable at the middle of both stator passages. Notice that the rotor wake is beginning to interact with the second stator wake at the hub region. Low background levels of turbulence (under 2\%) are encountered at midspan and central positions. These levels are gradually increased spanwise towards both casing and hub boundary layers, where maximum intensities are present. Nonetheless, viscous boundaries give no valuable information about the turbulence transport, so they have been removed from all the drawings to enhance the midspan colormap. At partial load (middle), there is a noticeable growth of the rotor wakes, as well as a major diffusion of the turbulent stator wakes in the background. The hub boundary layer is increased, as a consequence of a major flow separation in high-loaded zones of the blades. The scale of turbulence was also increased due to a higher level of disturbances. At near stall conditions (bottom), high levels of turbulence (even 20\%) are encountered within the rotor wakes.
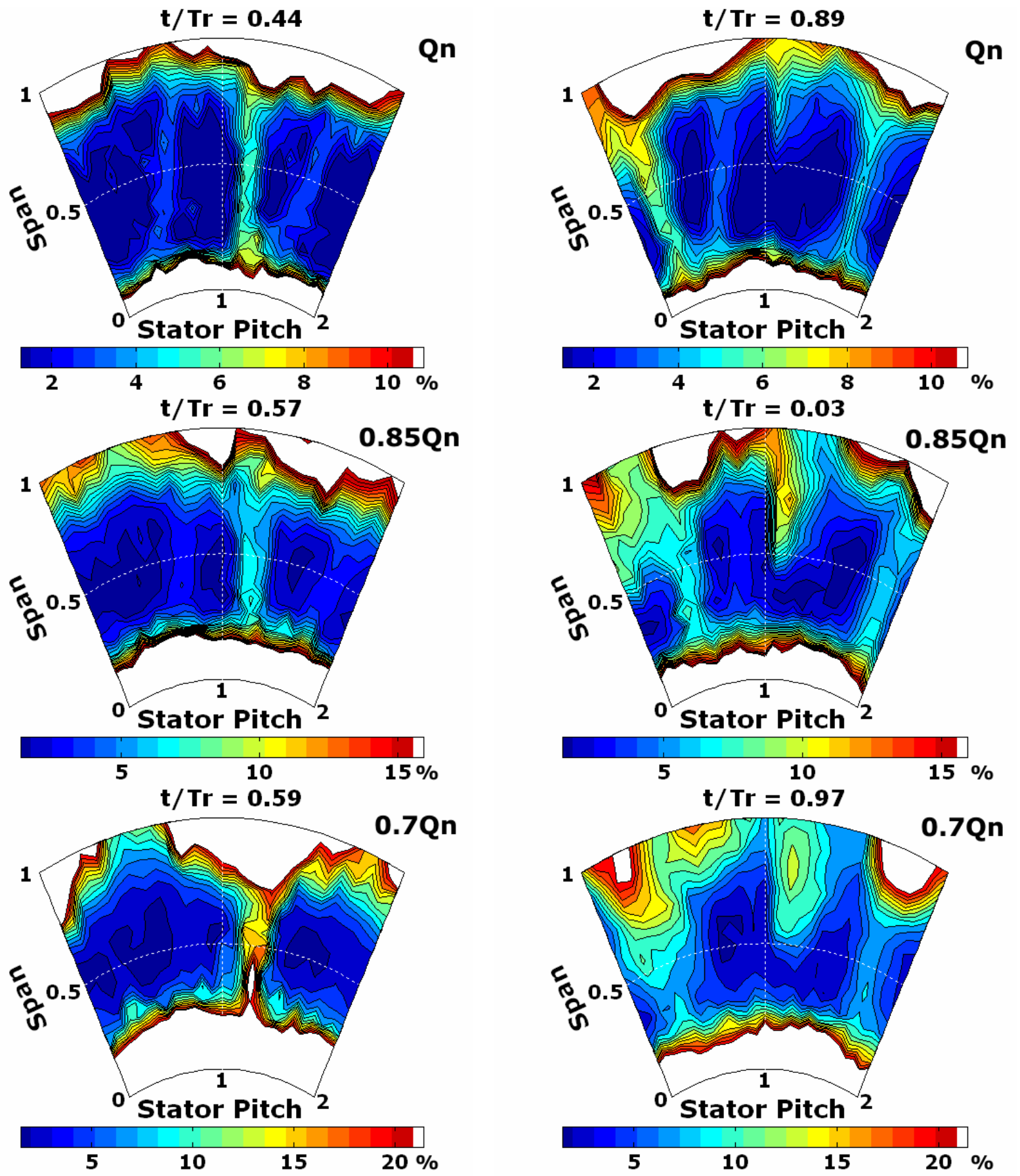

Fig. 16. Phase-averaged turbulence intensity downstream of the rotor for nominal conditions (top), partial load (middle) and near stall operating point (bottom). a) larger axial gap. b) lower axial gap. 
At this point, the stator wakes are practically diffused in the background and only a minor central band recalls periodic jet-wake structures from the blades.

Complementarily, figure $16 . \mathrm{b}$ (right column) presents similar results for the lower axial gap. In this case, all the operating conditions exhibit two rotor wakes in the drawings. This is possible because two stator pitches cover approx. $55.4^{\circ}$ and there are only $40^{\circ}$ between the rotor wakes. At nominal conditions (top), the stator wake appears more unmixed than before, because of the reduction in the axial gap of the stage. The second stator wake is now masked by a second rotor wake, whose intensity is clearly reduced spanwise with respect to the other rotor wake, as previously discussed in figure 15. Additionally, a new turbulent structure appears at the relative stator pitch position of 1.1. This kind of vortical disturbance suggests the existence of separated flow structures in the stator vanes. Though not shown here, this turbulent spot is stationary linked to that particular angular coordinate, instead of being transported tangentially. Therefore, its origin must be placed in the stator row despite being induced by the rotor blockage. Besides, if this phenomenon were simply developed in the stator and then convected across the stage by the mean-flow, the turbulence intensity should be of the order of the unmixed stator wakes. On the contrary, it presents similar levels to those transported by the rotor wakes, suggesting that this vortical structure is intensified when interacting with the rotor passage structures. At the moment, no satisfying explanation has been found by the authors to this characteristic of the flow. Both partial load (middle) and near stall conditions exhibit similar trends to those observed in figure 16.a. Basically, the dispersion of unmixed stator wakes increases, the rotor wakes broaden and overall disorder grows when the flow rate is reduced. The turbulent spot behaves like the other turbulent structures, enlarging its size and increasing its intensity.

The integral length scale at the rotor exit is influenced by the casing and hub boundary layers, by the unmixed dispersion of stator wakes and mainly by the periodic passing of rotor wakes. Its distribution is shown in figure 17 in the case of nominal operating conditions. In general, the overall range of large-scale eddies is drastically reduced from the stator exit, because of the continuous chopping mechanism of the rotor blades. Those large eddies (around 60-80 $\mathrm{mms}$ in figure 11) are broken up into smaller ones (15-20 mms) inside the rotor passages. On the other hand, the rotor wake fluid periodically crosses the measurement point and lowers the time-mean integral length scale (Henderson et al., [1]). Therefore, there is a remarkable zone, near the midspan, with minimum values of 10 mms, reflecting vortical disturbances of the rotor wakes. Also, the stator wakes are still present, with an integral length scale in the range of $25 \mathrm{mms}$. As the integral length scale increases with distance from the source of turbulence ([1]), it is coherent that eddies shed from the stator vanes shear layers evolve from $8 \mathrm{~mm}$ in Sector D (figure 11) to $25 \mathrm{~mm}$ downstream of the rotor. Both casing and hub viscous layers exhibit higher values of integral scale, which implies the creation of new eddies. In particular, the tip region can be influenced by tip vortices, whereas the hub section is clearly influenced by the shear layer between the separated region and the mainstream flow (Camp et al., [4]). Thus, figure 18 shows the spanwise displacement of the shear layer of the separation zone as the flow rate decreases. At nominal conditions, separation starts at a $20 \%$ of the midspan, while at near stall operating points, it has moved towards the midspan. Results in figure 18, obtained from pitch-averaging traverse maps, coincide with a radial increasing of the tangential velocity, analyzed in [15] (not shown here), which indicates the existence of separated flows in the hub.

The power spectrum of filtered fluctuating velocity at midspan indicates that a fully-developed turbulence has not been reached in the measurement location. Transport of turbulence by the unsteady rotor wakes is a continuous source of small eddies that have to redistribute their energy in the whole range of wavenumbers. Thus, the universal power-law in the inertial subrange is not followed by the experimental data in figure 19.

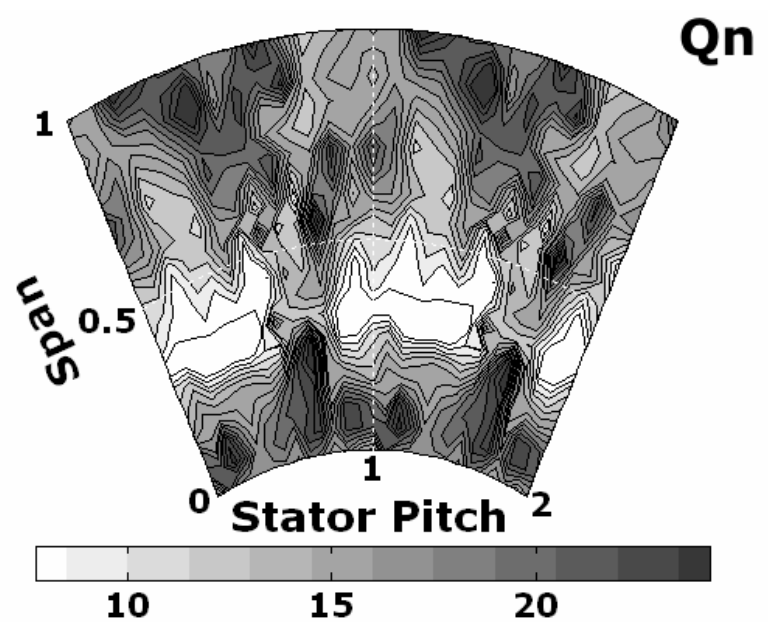

Fig. 17. Distribution of integral length scales (in $\mathbf{m m}$ ) at the rotor exit. Nominal conditions.

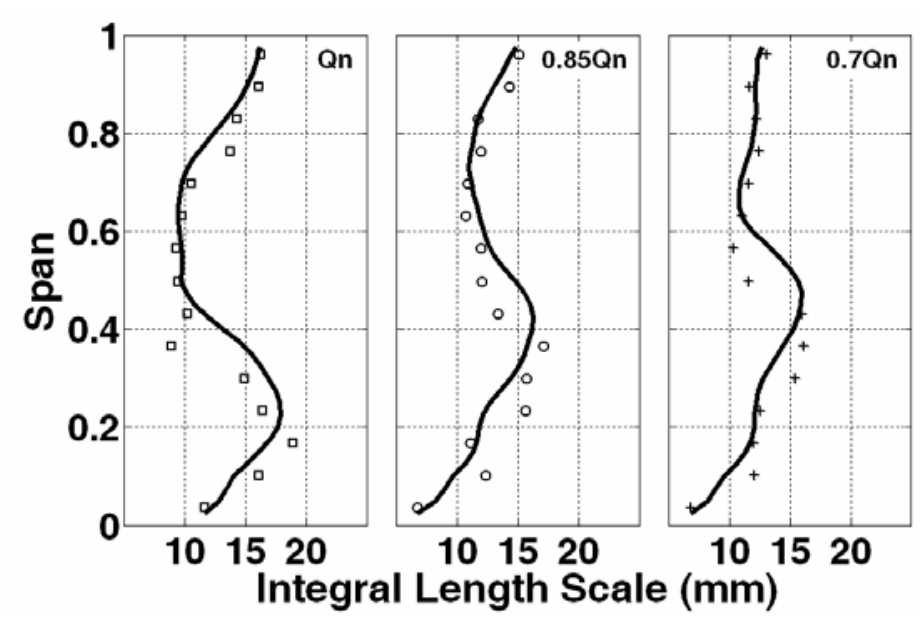

Fig. 18. Radial distribution of integral length scales (in mm) at the rotor exit. Influence of the operating point. 


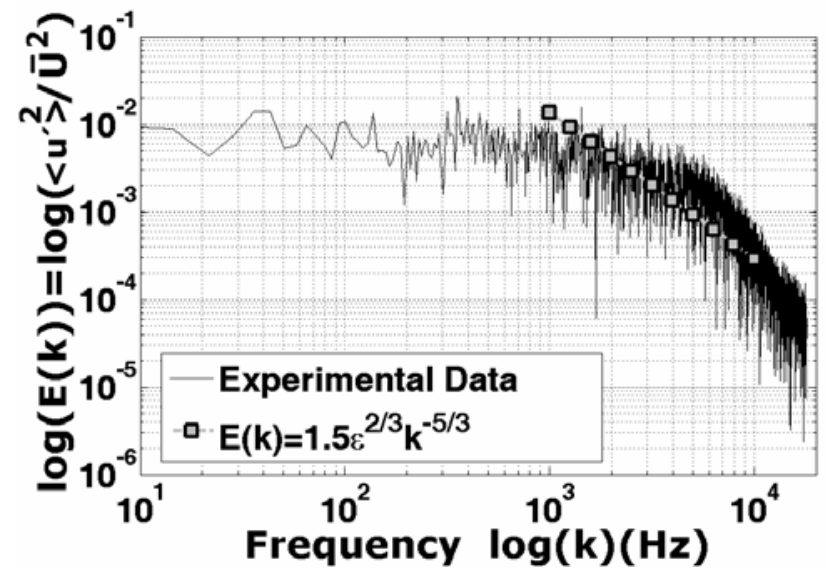

Fig. 19. Power spectrum of velocity fluctuations at midspan. Nominal conditions.

Turbulent Kinetic Energy Budget. The equation governing the ensemble-averaged kinetic energy, $\frac{1}{2} \widetilde{u_{i}^{\prime} u_{i}^{\prime}}$, of the turbulent velocity fluctuations is obtained by subtracting the ensemble-averaged RANS equations from the RANS equations. After some algebra, the final equation reads:

$$
\begin{gathered}
\left(\frac{\partial}{\partial t}+\tilde{U}_{j} \frac{\partial}{\partial x_{j}}\right)\left(\frac{1}{2} \widetilde{u_{i}^{\prime} u_{i}^{\prime}}\right)=-\frac{\partial}{\partial x_{j}}\left(\frac{1}{\rho} \widetilde{u_{j}^{\prime} p}+\frac{1}{2} \widetilde{u_{i}^{\prime} u_{i}^{\prime} u_{j}^{\prime}}\right) \\
-\frac{\partial}{\partial x_{j}}\left(-2 v \widetilde{u_{i}^{\prime} S_{i j}}\right)-\widetilde{u_{i}^{\prime} u_{j}^{\prime} S_{i j}}-2 v \widetilde{s_{i j} s_{i j}}
\end{gathered}
$$

Classical references ([13, 17]) employ timeaveraging, instead of ensemble-averaging, to describe the dynamics of the turbulent kinetic energy, considering that the mean flow has to be steady. With this constraint, deterministic phenomena are considered as total unsteadiness and the description of turbulence with periodic flow patterns is not feasible. In other words, since turbulence is not statistically steady in a multistage environment, time-averaging ignores the unsteady features of the flow. See Davidson - chapter 3[14] for a nice review of the need for statistical approaches and the different methods of taking averages that can be used when studying turbulence.

Equation (8) gives an exact idea of the rate of change in the turbulent kinetic energy (TKE) due to pressure-gradient work, transport by turbulent velocity fluctuations, transport by viscous stresses and two different deformation works: production and dissipation. Besides, the LHS of the equation separates both temporal and convective terms of the total variation of TKE. All the terms in the RHS explain the origin and the evolution of the changes in the TKE distribution, but it is difficult to determine them experimentally. They require extensive measurements for all the components in space due to the stress-tensor characteristics of the deformation works. This task is far beyond the scope of the present work. However, it is possible to determine in-plane distributions of the terms in the left-hand side from present experimental data.
Both temporal and convective terms, when expressed in cylindrical coordinates, take the following form:

$$
\begin{aligned}
& \frac{\partial q}{\partial t}+\left[\tilde{U}_{r} \frac{\partial q}{\partial r}+\frac{1}{r} \tilde{U}_{\theta} \frac{\partial q}{\partial \theta}+\tilde{U}_{z} \frac{\partial q}{\partial z}\right] \\
& q=\frac{1}{2}\left(\widetilde{u_{r}^{\prime} u_{r}^{\prime}}+\widetilde{u_{\theta}^{\prime} u_{\theta}^{\prime}}+\widetilde{u_{z}^{\prime} u_{z}^{\prime}}\right)
\end{aligned}
$$

In this case, the experiments provide only axial and tangential components of the velocity fluctuations in two traverse sectors. Hence, only radial and tangential derivatives can be computed. Of course, this is an important restriction, because out-of-plane (3D) effects may have an important impact on these terms, especially at hub and tip regions. Therefore, only an analysis of the midspan features has been attempted here, thus ignoring radial velocities. As a consequence, only the temporal and the $\theta$-component of the convective term will be discussed. In addition, TKE is computed using a $3 / 4$ coefficient, $q=\frac{3}{4}\left(\widetilde{u_{\theta}^{\prime} u_{\theta}^{\prime}}+\widetilde{u_{z}^{\prime} u_{z}^{\prime}}\right)$, to take into account the variance of the out-of-plane velocity component, assuming an average of the available measured components ([3]).

Figure 20 shows a temporal diagram with the evolution of the $\theta$-component of the convective term at the midspan of the stator exit. It has been normalized by the estimation of the dissipation rate, previously defined as $\varepsilon \sim u_{L}^{3} / L$. The importance of this term is clearly linked to the existence of averaged tangential velocities. At the stator exit, the major source of tangential components is placed at the vanes wakes. Positive bands in figure 20 indicate an increase of TKE when moving tangentially (entering the wake), while negative bands inform of the decrease of TKE along $\theta$ (leaving the wake). In addition, high positive and negative peaks appear in the bands, exhibiting the same pattern that was identified in figure 8 as the impact of the rotor blockage. But if the rotor blockage is a clear periodic, temporal fluctuation, why does its influence on the production of TKE appear in the convective term instead of that in the temporal one? The answer stems from the potential characteristic of this mechanism, which is modifying the averaged distribution of $U_{\theta}$ and convecting production of $q$ in the shear layers of the wakes. Actually, the rotor blockage is not inherently generating turbulence. As a consequence, in the distribution of $\partial q / \partial t$ (not shown here) no unsteady pattern related to BPF is observed and only small-scale turbulence contributes to the temporal term. In general, for nominal conditions, it can be assumed that the convective patterns present a higher order of magnitude than temporal ones. To conclude, it must be noticed that the impact of the rotor blockage in the convective term is more evident in case of lower axial gap. Also, lower flow rates are responsible for higher levels of global disturbance, so at off-design conditions, the temporal term becomes more important. 


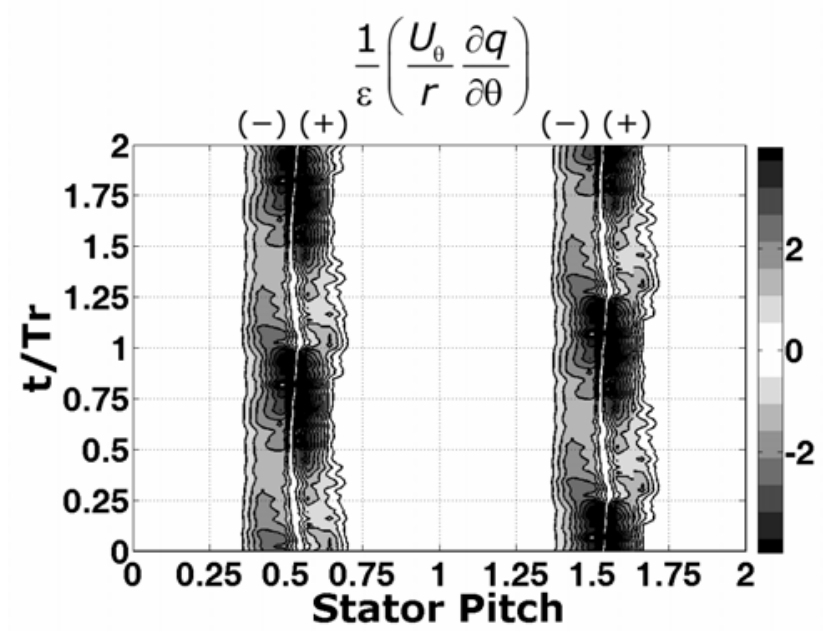

Fig. 20. Distribution of the convective term at the midspan between the rows at nominal conditions. Lower axial gap.

At the rotor exit, the balance between temporal and convective terms shows an opposite trend to that between the rows. In this case, the temporal term is more important than convective patterns (almost two orders of magnitude higher at nominal conditions, figure 21). $\partial q / \partial t$ represents the rate of change of TKE at a fixed point in space, which is exactly the temporal evolution acquired by the probe in the experiments. The smooth evolution of the rotor wakes has been obtained by pitch-averaging traverse map results. Then, it is assumed that dispersion of unmixed stator wakes has no influence on the generation of $q$. Effectively, in case of the convective term, the stator wake dispersion is not manifested as a deficit in the tangential velocity (only axial decay was observed in [15]), so it has no real contribution. Additionally, the stator wakes are steady in time, so every point in space is subject (or not) to the presence of the wakes in the same way over time. Thus, they cannot be responsible for temporal variations of TKE.

The influence of flow rate conditions on both terms is analyzed in figure 21 . On the left, the temporal term addresses the broadening of rotor wakes when the flow rate is reduced. At nominal conditions (thicker solid line), high and narrow oscillations in positive and negative zones indicate the presence of thin shear layers at both SS and PS. At partial load and near stall, important reductions of the fluctuations indicate the establishment of lower gradients, i.e., thicker boundary layers. On the right, the convective term enlarges as the flow rate decreases. It is derived from the progressive underturning of the flow, leading to the generation of secondary flow at the rotor exit. Therefore, as the flow rate decreases, the secondary tangential velocity enhances the convection term.

\section{CONCLUSIONS}

The structure of turbulence in a single stage, lowspeed axial fan with inlet guide vanes has been analyzed in detail. Both turbulent intensities and integral length
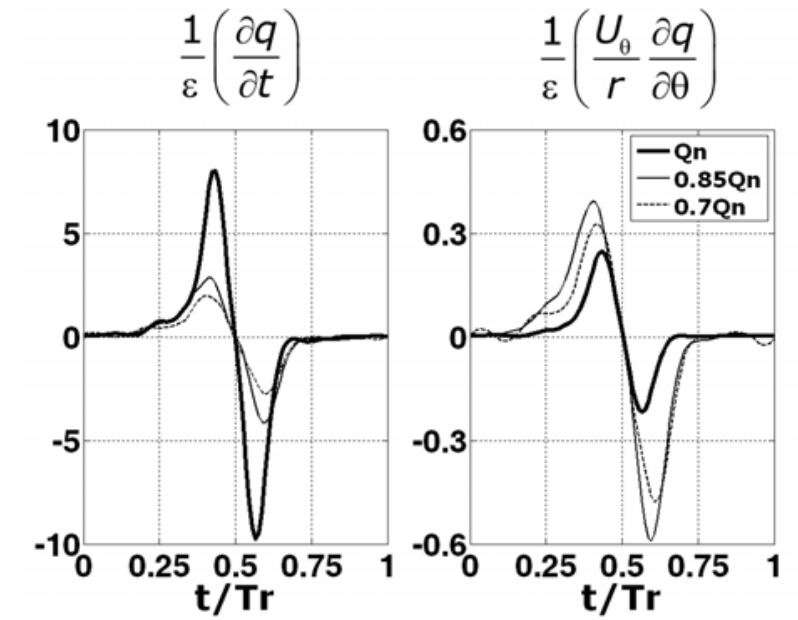

Fig. 21. Distribution of both convective and temporal terms at midspan rotor downstream as a function of the mass flow rate.

scales have been measured across the stage using hotwire techniques intensively. In order to decouple periodic, unsteady flow patterns from random turbulence, it was necessary to develop a processing data method -based on FFT filtering-, to remove all the periodicity in the velocity traces. Additionally, ensemble-averaging was used to highlight the transport of the turbulent structures caused by the unsteady flow patterns. Studying the influence of other parameters, like the variation in the flow rate, or the impact of the axial gap in the turbulent stator-rotor interaction, it was possible to obtain a complete picture of the flow turbulence.

The results of turbulence intensities and integral length scales have shown good agreement with other data reported in the literature in the case of similar lowspeed axial compressors. The estimation of those length scales across the stage provides useful data to define good practices for the numerical discretization of CFD modeling of axial fans. Also, the existence of high-order periodicities, manifested as large-scale fluctuations in the spectra, has demonstrated that additional modeling of these unsteady sources may be as necessary as regular modeling of chaotic turbulence.

At the stator exit, the impact of the rotor blockage on the shear layers of the stator wakes has been identified as a source of TKE production, and therefore major loss generation. Analysis of in-plane terms of the LHS of the TKE transport equation provided further insight on the mechanisms of generation. At the rotor exit, the reduction of mass flow rate was shown to have a significant influence in the wake dispersion and the stator wake-rotor wake interaction. The stator wake fluid was more widely diffused and reduced values of the stator wake velocity deficit were found when compared to midpassage positions. Complementarily, traverse maps of phase-averaged turbulence have perfectly illustrated the characteristics of all the turbulent phenomena involved in the stage, describing the complexity of the unsteady transport of chaotic randomness. 


\section{ACKNOWLEDGEMENTS}

This work was supported by the Research Project "Modeling of Deterministic Stresses in Axial Turbomachinery", ref. DPI2003-09712, CICYT.

\section{NOMENCLATURE}

$\begin{array}{ll}\text { ACF } & \text { Autocorrelation function. } \\ \text { B } & \text { Number of rotor blades. } \\ \text { BPF } & \text { Blade passing frequency, }\left[\mathrm{s}^{-1}\right] . \\ E & \text { Spectrum of turbulent energy, }\left[\mathrm{m}^{2} / \mathrm{s}^{2}\right] . \\ \text { FFT } & \text { Fast Fourier Transform. } \\ k & \text { Frequency or eddies wavenumber, }\left[\mathrm{s}^{-1}\right] . \\ L & \text { Integral length scale, }[\mathrm{m}] . \\ \text { LE } & \text { Leading edge. } \\ M & \text { Total number of stored rotor blades periods, }[-] . \\ N & \text { Number of samples per blade passing period, }[-] . \\ P & \text { Pressure, [Pa]. } \\ \mathrm{PS} & \text { Pressure side. } \\ \mathrm{Q}, \mathrm{Qn} & \text { Flow rate and nominal flow rate, }\left[\mathrm{m}^{3} / \mathrm{s}\right] . \\ q & \text { Turbulent kinetic energy, }\left[\mathrm{m}^{2} / \mathrm{s}^{2}\right] . \\ \vec{r} & \text { Spatial position in cylindrical coordinates. } \\ r & \text { Radial coordinate, }[\mathrm{m}] . \\ \mathrm{Re} & \text { Reference Reynolds number, }[-] . \\ \mathrm{SS} & \text { Suction side. } \\ t & \text { Time, }[\mathrm{s}] . \\ T_{r} & \text { Rotor blade passing period, }[\mathrm{s}] . \\ T u & \text { Turbulence intensity, }[\%] . \\ \text { TE } & \text { Trailing edge. } \\ \mathrm{TKE} & \text { Turbulent Kinetic Energy. } \\ u & \text { Instantaneous velocity, }[\mathrm{m} / \mathrm{s}] . \\ u^{\prime} & \text { Random velocity fluctuation, }[\mathrm{m} / \mathrm{s}] . \\ u & \text { Characteristic velocity scale of largest eddies, } \\ & \text { [m/s]. } \\ \tilde{U} & \text { Deterministic velocity component, }[\mathrm{m} / \mathrm{s}] . \\ U & \text { Deterministic velocity fluctuation, }[\mathrm{m} / \mathrm{s}] . \\ \bar{U} & \text { Mean velocity, [m/s]. } \\ & \text { Axial coordinate, [m]. } \\ & \end{array}$

\section{Greek letters}

$\begin{array}{cl}\alpha & \text { Kolmogorov's constant, [-]. } \\ \varepsilon & \text { Energy dissipation rate, }\left[\mathrm{m}^{2} / \mathrm{s}^{3}\right] . \\ \phi & \text { Flow coefficient, [-]. } \\ \varphi & \text { Rotor blade phase, }\left[{ }^{\circ}\right] . \\ \eta & \text { Overall efficiency, [-]. } \\ \theta & \text { Angular coordinate, [rad]. } \\ \vartheta & \text { Rotor phase, }\left[^{\circ}\right] . \\ \rho & \text { Density, }\left[\mathrm{kg} / \mathrm{m}^{3}\right] . \\ \tau & \text { Time lag, }[\mathrm{s}] . \\ \psi & \text { Pressure coefficient, }[-] . \\ \Omega & \text { Rotational speed, }[\mathrm{rad} / \mathrm{s}] .\end{array}$

\section{Superscripts and Subscripts}

$\begin{array}{ll}<> & \text { FFT filtering. } \\ \sim & \text { Ensemble-averaging. } \\ - & \text { Time-averaging. } \\ & \text { Tip . } \\ t & \text { Inlet and outlet. }\end{array}$

\section{REFERENCES}

[1] Henderson, A.D., Walker, G., Hughes, J.D., 2006, The Influence of Turbulence on Wake Dispersion and Blade Row Interaction in an Axial Compressor, ASME Journal of Turbomachinery, Vol. 152, pp. 150-157.

[2] Mailach, R., Vogeler, K., 2004, Aerodynamic Blade Row Interactions in an Axial Compressor - Part I: Unsteady Boundary Layer Development, ASME Journal of Turbomachinery, Vol. 126, pp. 35-44.

[3] Uzol, O., Chow, Y-C., Katz, J., Meneveau, C., 2002, Experimental Investigation of Unsteady Flow Field Within a Two-Stage Axial Turbomachine Using Particle Image Velocimetry, ASME Journal of Fluids Engineering, Vol. 124, pp. 542-552.

[4] Camp, T.R., Shin, H.-W., 1995, Turbulence Intensity and Length Scale Measurements in Multistage Compressors, ASME Journal of Turbomachinery, Vol. 117, pp. 38-46.

[5] Cherret, M.A., Bryce, J.D., 1992, Unsteady Viscous Flow in High-Speed Core Compressor, ASME Journal of Turbomachinery, Vol. 114, pp. 287-294.

[6] Goto, A., 1992, Three-Dimensional Flow and Mixing in an Axial Flow Compressor With Different Rotor Tip Clearances, ASME Journal of Turbomachinery, Vol. 114, pp. 675-685.

[7] Senkter, A., Riess, W., 1998, Measurement of Unsteady Flow and Turbulence in a Low Speed Axial Compressor, Experimental Thermal and Fluid Science, Vol. 17, pp. 124-131.

[8] Senkter, A., Riess, W., 2000, Experimental Investigation of Turbulent Wake-Blade Interaction in Axial Compressors, International Journal of Heat and Fluid Flow, Vol. 21, pp. 285-290.

[9] Ballesteros, R., Velarde, S., Santolaria, C., 2002, Turbulence Intensity Measurements in a Forward-Curved Blades Centrifugal Fan, Proceedings of the XXIst IAHR Symposium on Hydraulic Machinery and Systems, Lausanne, 9-12 Sept.

[10] Velarde-Suárez, S., Ballesteros-Tajadura, R., SantolariaMorros, C., Blanco-Marigorta, E., 2002, Total Unsteadiness Downstream of an Axial Flow Fan With Variable Pitch Blades, ASME Journal of Fluids Engineering, Vol. 124, pp. 280-283.

[11] Huyer, S.A., Snarski, S.R., 2003, Analysis of a Turbulent Propeller Inflow, ASME Journal of Fluids Engineering, Vol. 123, pp. 533-542.

[12] Blanco E., Ballesteros, R., Santolaria, C., 1998, Angular Range and Uncertainty Analysis of Non-Orthogonal Crossed Hot Wire Probes, ASME Journal of Fluids Engineering, Vol. 120, pp.90-94.

[13] Tennekes, H., Lumley, J.L., 1972, A First Course in Turbulence, The MIT Press.

[14] Davidson, P.A., 2004, Turbulence: an Introduction for Scientists and Engineers, Oxford University Press.

[15] Fernández Oro, J.M., 2005, Unsteady Rotor-Stator Interaction in an Axial Turbomachine, Ph.D. Thesis, University of Oviedo, Spain.

[16] Fernández Oro, J.M., Argüelles Díaz, K.M., Santolaria Morros, C., Blanco Marigorta, E., 2005, Unsteady RotorStator Interaction in an Axial Flow Blower. Part I: Numerical and Experimental Flow Field Characterization, Proceedings of the 2005 ASME Fluids Engineering Summer Conference, Houston, TX-USA.

[17] McComb, W.D., 1990, The Physics of Fluid Turbulence, Oxford University Press. 
This document is a pre-print version of the scientific paper published by Elsevier. It has been released by the authors to fulfill all the publisher requirements established for Article Sharing: https://www.elsevier.com/about/policies/sharing

\section{(9) $\Theta \Theta \Theta$}

(C) 2019. This manuscript version is made available under the Creative Commons Attribution-NonCommercial-NoDerivatives 4.0 International License (CC-BY-NC-ND 4.0 license) http://creativecommons.org/licenses/by-nc-nd/4.0/ 